Florida International University FIU Digital Commons

\title{
The Role of Temporal Synchrony in the Facilitation of Perceptual Learning during Prenatal Development
}

Mark Jaime

Florida International University, mrk_jaime@yahoo.com

DOI: $10.25148 /$ etd.FI08081526

Follow this and additional works at: https://digitalcommons.fiu.edu/etd

\section{Recommended Citation}

Jaime, Mark, "The Role of Temporal Synchrony in the Facilitation of Perceptual Learning during Prenatal Development" (2007). FIU Electronic Theses and Dissertations. 58.

https://digitalcommons.fiu.edu/etd/58 


\section{FLORIDA INTERNATIONAL UNIVERSITY}

Miami, Florida

THE ROLE OF TEMPORAL SYNCHRONY IN THE FACILITATION OF PERCEPTUAL LEARNING DURING PRENATAL DEVELOPMENT

A dissertation submitted in partial fulfillment of the

requirements for the degree of

DOCTOR OF PHILOSOPHY

in

PSYCHOLOGY

by

Mark Jaime

2007 
To: Interim Dean Mark Szuchman

College of Arts and Sciences

This dissertation, written by Mark Jaime, and entitled The Role of Temporal Synchrony in the Facilitation of Perceptual Learning during Prenatal Development, having been approved in respect to style and intellectual content, is referred to you for judgment.

We have read this dissertation and recommend that it be approved.

Lorraine Bahrick

Lidia Kos

Mary Levitt

David Lewkowicz

Robert Lickliter, Major Professor

Date of Defense: November 7, 2007

The dissertation of Mark Jaime is approved.

Interim Dean Mark Szuchman

College of Arts and Sciences

Dean George Walker

University Graduate School

Florida International University, 2007 


\section{DEDICATION}

This dissertation is dedicated to my parents, Ana Jaime and Fred Jaime, for this is

their accomplishment as much as it is mine. Without their loving support this would not be possible. I am proud to be their son. 


\section{ACKNOWLEDGMENTS}

First and foremost, I wish to thank Dr. Robert Lickliter. He has been an exceptional mentor, model scholar, and friend. I am forever grateful for all of his support and guidance. I also wish to thank the members of my committee for showing genuine interest in making this dissertation the best it could possibly be. Finally, I wish to thank Dr. Charles Bigger, Esther Lopez, and the MBRS RISE program for providing the financial support that has fostered and enhanced my research interests and goals. 


\title{
ABSTRACT OF THE DISSERTATION \\ THE ROLE OF TEMPORAL SYNCHRONY IN THE FACILITATION OF \\ PERCEPTUAL LEARNING DURING PRENATAL DEVELOPMENT
}

\author{
by
}

Mark Jaime

Florida International University, 2007

Miami, Florida

\section{Professor Robert Lickliter, Major Professor}

This study explored the critical features of temporal synchrony for the facilitation of prenatal perceptual learning with respect to unimodal stimulation using an animal model, the bobwhite quail. The following related hypotheses were examined: (1) the availability of temporal synchrony is a critical feature to facilitate prenatal perceptual learning, (2) a single temporally synchronous note is sufficient to facilitate prenatal perceptual learning, with respect to unimodal stimulation, and (3) in situations where embryos are exposed to a single temporally synchronous note, facilitated perceptual learning, with respect to unimodal stimulation, will be optimal when the temporally synchronous note occurs at the onset of the stimulation bout.

To assess these hypotheses, two experiments were conducted in which quail embryos were exposed to various audio-visual configurations of a bobwhite maternal call and tested at $24 \mathrm{hr}$ after hatching for evidence of facilitated prenatal perceptual learning with respect to unimodal stimulation. Experiment 1 explored if intermodal equivalence was sufficient to facilitate prenatal perceptual learning with respect to unimodal stimulation. A Bimodal Sequential Temporal Equivalence (BSTE) condition was created 
that provided embryos with sequential auditory and visual stimulation in which the same amodal properties (rate, duration, rhythm) were made available across modalities. Experiment 2 assessed: (a) whether a limited number of temporally synchronous notes are sufficient for facilitated prenatal perceptual learning with respect to unimodal stimulation, and (b) whether there is a relationship between timing of occurrence of a temporally synchronous note and the facilitation of prenatal perceptual learning.

Results revealed that prenatal exposure to BSTE was not sufficient to facilitate perceptual learning. In contrast, a maternal call that contained a single temporally synchronous note was sufficient to facilitate embryos' prenatal perceptual learning with respect to unimodal stimulation. Furthermore, the most salient prenatal condition was that which contained the synchronous note at the onset of the call burst. Embryos' prenatal perceptual learning of the call was four times faster in this condition than when exposed to a unimodal call. Taken together, bobwhite quail embryos' remarkable sensitivity to temporal synchrony suggests that this amodal property plays a key role in attention and learning during prenatal development. 


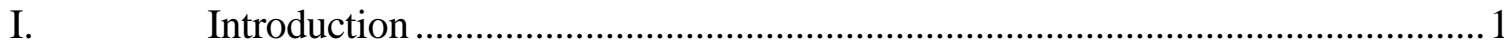

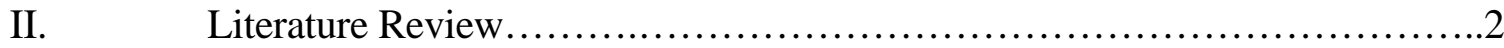

Infant Responsiveness to Temporal Synchrony..............................2

The Effect of Audio-Visual Temporal Synchrony on Attention:

Neurophysiologic and Behavioral Consequences...............................5

The Effects of Intersensory Redundancy on Attention, Learning, and

Memory during Early Development .................................................................... 11

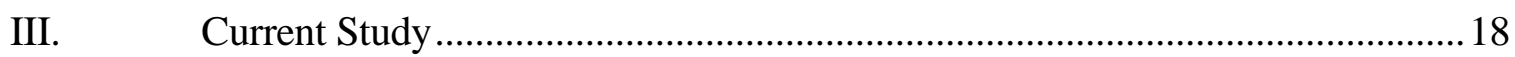

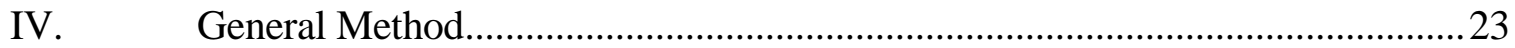

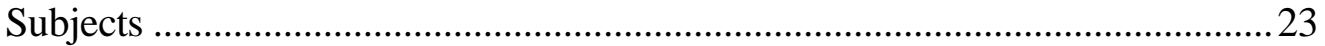

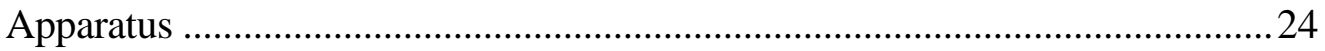

Procedure................................................................................................. 25

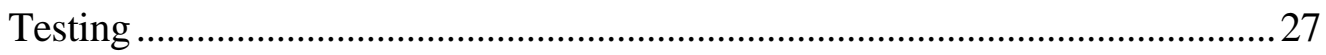

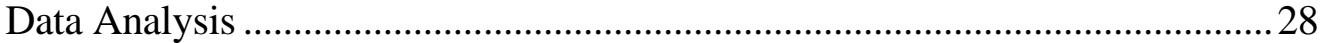

V. Experiment 1: Temporal Synchrony as a Critical Feature for the Facilitation of Prenatal Perceptual Learning.............................................29

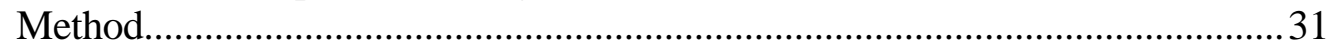

Results and Discussion ....................................................................................... 33

VI. Experiment 2a: Exploring the Sufficient Features for the Facilitation of Prenatal Perceptual Learning: Amount of Temporally Synchronous Notes.....39

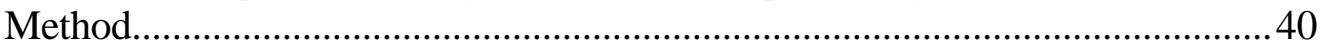

Results and Discussion .................................................................................... 41

VII. Experiment 2b: Exploring the Sufficient Features for the Facilitation of Prenatal Perceptual Learning: Timing of Temporally Synchronous Notes........46

Method............................................................................................................4

Results and Discussion .................................................................................. 47

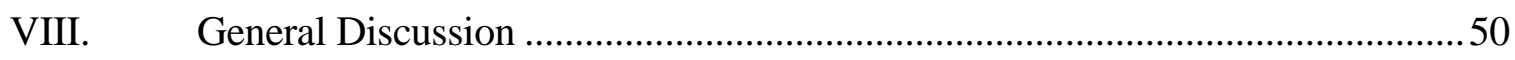

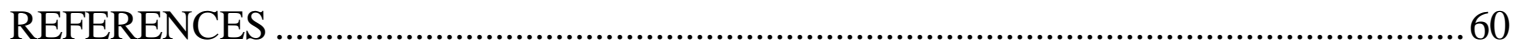

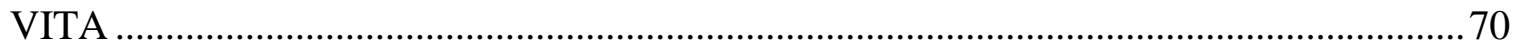




\section{LIST OF TABLES}

TABLE

PAGE

1. Mean Proportions of Total Duration Time and Standard Deviations (in seconds) for

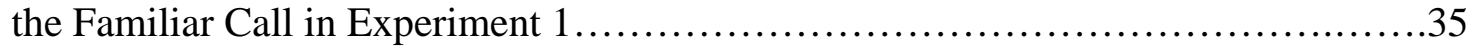

2. Means and Standard Deviations of Duration Scores (in seconds) for Experiment 1....36

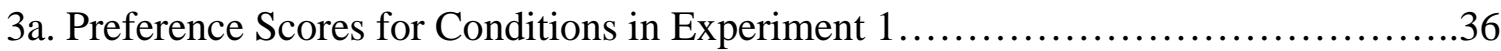

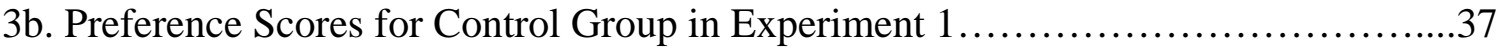

4. Mean Proportions of Total Duration Time and Standard Deviations (in seconds) for

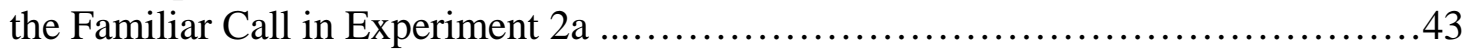

5. Means and Standard Deviations of Duration Scores (in seconds) for Experiment 2a ..43

6. Preference Scores for Conditions in Experiment 2a.............................44

7. Mean Proportions of Total Durations Time and Standard Deviations (in seconds) for the Familiar Call in Experiment $2 b$..............................................48

8. Means and Standard Deviations of Duration Scores (in seconds) for Experiment 2b...49

9. Preference Scores for Conditions in Experiment 2b. 


\section{LIST OF FIGURES}

FIGURE

PAGE

1. Top View of the Behavioral Testing Arena.....................................25

2. Spectrograms of the Bobwhite Maternal Calls (A and B)..........................26

3. Schematic Representation of the Various Audio-Visual Configurations of the

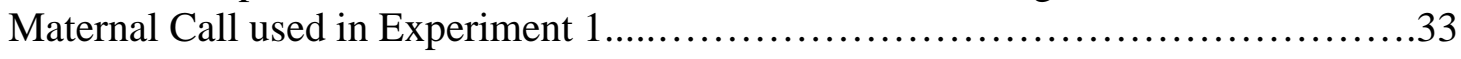

4. Mean Proportion of Total Duration Time for the Familiar Call in Experiment 1.......34

5. Schematic Representation of the Various Audio-Visual Configurations of the Maternal Call used in Experiment 2a.......................................41

6. Mean Proportion of Total Duration Time for the Familiar Call in Experiment 2a......42

7. Mean Proportion of Total Duration Time for the Familiar Call in Experiment 2b.......48 
Introduction

Organisms develop in a world containing temporally coordinated multimodal events. As a result, animal and human infants acquire a considerable amount of experience with temporally synchronous multisensory stimulation. The prenatal environment also provides temporally synchronous patterns of vestibular, auditory, and visual stimulation. For example, a fetus can experience the synchrony between its mother's vocalizations and her bodily vibrations. Temporal synchrony likely plays an important role in adaptive functioning by providing redundant information about the unity and location of multimodal events. Comparative studies have demonstrated that orientation to an audio-visual stimulus is facilitated when the auditory and visual cues occur in temporal synchrony (e.g., Jiang, Jiang, \& Stein, 2002; Stein, Meredith, Huneycutt, \& McDade, 1989; Wilkinson, Meredith, \& Stein, 1996). Furthermore, sensitivity to temporal synchrony has been proposed to serve as the basis for the development of higher order cognitive processes (Edelman, 1992; Gibson, 1969; Thelen \& Smith, 1994).

The detection of temporal properties appears to follow a hierarchical order during early infant development such that temporal properties containing global characteristics are detected first. However, as infants gain experience, they can detect temporal properties characterized by specific or arbitrary relations (Bahrick, 1992, 2001). Temporal synchrony is considered to be the most global temporal property because it provides relational information about the sights and sounds of multimodal events (Bahrick, 2001; Lewkowicz, 2000) and is the earliest temporal property detected by human infants (e.g., Bahrick, 2001; Slater, Quinn, Brown, \& Hayes, 1999). The ability 
for infants to detect other temporal properties such as duration (Lewkowicz, 1986), rate (e.g., Lewkowicz, 1986, 1988, 1992; Spelke, 1979), and rhythm (e.g., Mendelson \& Ferland, 1982; Pickens \& Bahrick, 1995, 1997) appears to emerge subsequent to temporal synchrony detection. However, it is important to note that the emergence of sensitivity to various temporal properties is not linear. That is, sensitivity to one temporal property may aid in the detection of other properties throughout perceptual development. This study focuses on the detection of temporal synchrony and its role in early perceptual learning. This study demonstrates that temporal synchrony plays a primary role in organizing learning and attentional systems during prenatal development by the embryo's remarkable facilitated perceptual learning of synchronous stimulation with respect to learning unimodal prenatal stimulation.

\section{Literature Review \\ Infant Responsiveness to Temporal Synchrony}

Developing infants are exposed to an array of moving sights and sounds. How do infants come to know which sights and sounds are related and which are not? Infants' early sensitivity to temporal synchrony facilitates veridical intersensory perception. For example, intermodal preference studies have consistently demonstrated that temporal synchrony facilitates in the forming of audio-visual associations of object-sound pairings in newborns (Morrongiello, Fenwick, \& Chance, 1998; Slater, Quinn, Brown, \& Hayes, 1999) and young infants (e.g., Bahrick, 1987, 1988; Lawson, 1980; Lyons-Ruth, 1977; Spelke, 1979). Temporal synchrony affords infants the opportunity to learn that synchronized sights and sounds "belong together" rather than forming inappropriate associations in the vast array of available sights and sounds. 
Infants' initial affinity towards the amodal property of temporal synchrony also plays a role in the coordination of attention (Lewkowicz, 2000; Lickliter \& Bahrick, 2000). This is best exemplified in a study conducted by Bahrick and colleagues. Bahrick, Walker, and Neisser (1981) presented 4-month-old infants with two superimposed videos and a soundtrack that corresponded to only one of the videos. Results demonstrated that infants were able to focus their attention on the image that was synchronized to the soundtrack. Temporal synchrony also provides the infant perceiver with information about specific temporally-based stimulus properties. Infants are also sensitive to the temporal synchrony of sound and visible impact specifying object substance (Bahrick, 1983) and composition (Bahrick, 1987, 2001). For example, Bahrick (1987) presented 3, 4 1/2, and 6-month-old infants with two side-by-side films of a transparent cylinder, one containing a single large marble and the other transparent cylinder containing several smaller marbles. Both cylinders were abruptly turned back and forth in an erratic pattern, creating a clear impact sound with each turn while the sound matching only one of the videos that emanated from a central location between the two. Results demonstrated that the 6-month-old infants matched the moving objects and sounds on the basis of the temporal micro-structure specifying single versus compound objects.

This same developmental trend was observed in a study with much younger infants. Using an infant controlled habituation procedure, Bahrick (2001) demonstrated developmental trends in 4, 7, and 11-week-old infants' sensitivity to temporal synchrony and temporal microstructure specifying object composition. Results revealed that 4week-old infants detected global temporal synchrony relations but not temporal 
microstructure specifying object composition. However, detection of temporal microstructure was evident in 7- and 11-week-old infants. In accord with Bahrick's previous findings (Bahrick, 1987), older infants are better at detecting more specific, nested, levels of temporal information of multimodal events. These results provide evidence that the early development of intersensory perception proceeds from detecting global properties of multisensory events, such as temporal synchrony, to more specific properties such as temporal micro-structure. Thus, experience makes infants more skilled perceivers of specific temporal properties.

Further evidence highlighting the importance of experience on increasing sensitivity to temporal properties comes from studies on the Intersensory Temporal Contiguity Window (ITCW) of audio-visual stimulation (Lewkowicz, 1996, 2000). The ITCW is the minimum length of time (in milliseconds) between which an audible and visible stimulus must occur in order for one to perceive the event as synchronous. This concept of a temporal window for the perception of temporal synchrony has been previously observed in adults. For adults, the length of the ITCW varies depending on the order in which the auditory and visual stimuli are presented (Fraisse, 1982). For example, studies have demonstrated that adults can perceive a bouncing audio-visual event as temporally synchronous with an ITCW of approximately 65-80 milliseconds, provided the sound comes before the visible bounce and 112-140 milliseconds if the sound follows the visible bounce (see Lewkowicz, 1996; McGrath \& Summerfield, 1985; Summerfield, 1979).

In infants, however, the ITCW is significantly larger, regardless of the order of the sound presentation. Lewkowicz (1996) demonstrated that infants have a greater 
tolerance for temporal disparity in their perception of synchrony. Specifically, infants at 2 and 8 months of age were tested using an infant-controlled habituation procedure. In this experiment, infants were first habituated to an audio-visual presentation of a green disk moving up and down on a TV monitor. A sound was presented at the instant the moving disc changed its direction at the bottom of the screen. Thus, the synchronous presentation of a sound with a change in direction gave infants the illusion of a bouncing effect. Subsequent test trials consisted of a change in the timing of the synchrony between the sound and the visible impact. Results demonstrated that the ITCW for infants is approximately 350 milliseconds, a much wider ITCW than observed in adults. These results suggest that infants have a greater tolerance for audio-visual temporal disparity and they perceive relatively large disparities (when compared to adults) as synchronous. This insight raises the interesting question of what constitutes "effective" synchrony in terms of guiding early perceptual learning.

The Effect of Audio-Visual Temporal Synchrony on Attention: Neurophysiologic and Behavioral Consequences

The superior colliculus (SC) is an area of the midbrain composed of multisensory neurons responsible for initiating attentive and orientation-like behaviors in a variety of species (e.g., Casagrande, Harting, Hall, \& Diamond, 1972; Jay \& Sparks, 1984; Meredith \& Stein, 1983, 1986a; Schneider, 1967; Sprague \& Meikle, 1965; Stein \& Meredith, 1991; Wallace, Wilkenson, \& Stein, 1996). Research investigating the behavior of these neurons has provided evidence demonstrating that temporally synchronous audio-visual stimulation produces enhanced neural responses that are greater than the sum of each individual unimodal component (see Stein \& Meredith, 
1993, for a review). This "multiplicative" or "superadditive" effect has been demonstrated across a variety of extracellular recording techniques, including response reliability, decreased reaction time, impulses evoked, peak impulse frequency, and duration of discharge (reviewed in Stein \& Meredith, 1993; Stein, Meredith, \& Wallace, 1993). This effect is part of a system in which neural responsiveness in the SC depends on the temporal and spatial parameters of sensory inputs.

This system is composed of two basic types of neural influences in the SC, response enhancement and response depression (King \& Palmer, 1985; Meredith \& Stein, 1983; 1986a, 1986b; Wallace, Meredith, \& Stein, 1998; Wallace, Wilkinson, \& Stein, 1996). They are respectively defined as the augmentation or diminution of a neural response to a stimulus of one modality by the presentation of a second stimulus from another modality and are affected by the temporal relationship between auditory and visual stimuli (Kadunce, Vaughan, Wallace, Benedek, \& Stein, 1997; Meredith, Nemitz, \& Stein, 1987; Meredith \& Stein, 1986a, 1996). For example, temporally synchronous auditory and visual events produce response enhancement, while temporally asynchronous auditory and visual stimulus events result in response depression. Such response enhancement and depression mechanisms ensure that organisms can selectively attend to unitized stimulus events without being distracted by other sensory stimulation in the immediate surround.

Multisensory enhancement and/or depression are not generalized effects of SC neurons; rather, they occur because the topographical distributions of auditory and visual neurons in the SC are in spatial register with one another. In other words, the auditory and visual unimodal receptive fields overlap. Response enhancement and/or depression 
occur under specific spatio-temporal rules. For example, a visual stimulus that occurs within the auditory receptive field enhances auditory neural responsiveness in that area. In contrast, if a visual stimulus occurs outside the auditory receptive field, it will fail to enhance responsiveness of the auditory neurons and significantly depresses their response. These receptive fields are arranged so that a multisensory event located directly in front of the organism will produce the greatest neural interactions while those stimulus events occurring at the periphery of the midline produce response depression. This spatial rule makes sense when one considers that the best way to locate a stimulus event among many is to orient the head so that the stimulus event is closest to the midline. Because of the fact that multisensory events are usually temporally and spatially contiguous, orienting to a stimulus event so that it aligns closest to midline allows the perceiver to optimally detect the amodal temporal and spatial properties that specify its unity.

In addition to spatial rules, there are also important temporal rules that are at play in multisensory interactions of SC neurons. Although counterintuitive, the most intense interactions are not necessarily produced by matching physical onsets and latencies; instead, interactions can occur at any time within a specified temporal window. The duration of the temporal window depends on the duration of discharges a stimulus evokes. Temporal windows have been observed to range from $250 \mathrm{msec}$ for visualsomatosensory neurons, to $1500 \mathrm{msec}$ for some visual-auditory neurons. For example, if an auditory stimulus invokes a series of discharges lasting $300 \mathrm{msec}$, a visual stimulus occurring within this $300 \mathrm{msec}$ period will create an interaction. This interaction can, in 
turn, induce an additional train of discharges thus extending the duration of the temporal window.

Another key factor for response enhancement is the overlap of the peak discharge pattern of the stimuli. For example, a $300 \mathrm{msec}$ auditory stimulus that contains its peak discharge patterns at $250 \mathrm{msec}$ will produce the most intense enhancement effect from a visual stimulus if it occurs at $250 \mathrm{msec}$. Since sensory stimulation energies travel at different speeds (e.g., light $=186,000 \mathrm{ft} / \mathrm{sec}$, sound $=1100 \mathrm{ft} / \mathrm{sec}$ ), the temporal window allows the possibility of an interaction with later arriving stimuli. This relatively wide window of time provides the organism more opportunity to detect subtle yet potentially important stimuli at varying distances and plays an integral role in orientation and localization (Stein, Huneycutt, \& Meredith, 1988; Stein, Meredith, Huneycutt, \& McDade, 1989). For example, a predator stalking its prey in a dense forest uses visual, auditory, and olfactory cues to locate its prey. If the prey is at a considerable distance from the predator the different sensory cues will reach its sensory receptors at varying times. If the temporal window is too narrow, there would be little opportunity for neural enhancement mechanisms to take effect and aid in localizing the prey. But when the temporal window is wide enough there is greater probability of neural enhancement, which leads to facilitated attention, orientation, and ultimately success at catching the prey.

Previous comparative research on multisensory responses in the superior colliculus has involved experiments with cats, a highly altricial species (Stein \& Meredith, 1993). When cats are born, their visual system is not completely developed. In contrast, precocial birds are born with all of their sensory systems functional. 
Although there is little neurophysiologic research on multisensory interactions in the embryonic brain of precocial birds, investigations on the prenatal development of the avian auditory and visual systems have been performed (e.g., Mey \& Thanos, 1993; Momose-Sato, Glover, \& Sato, 2006). For example, studies using single-unit recordings from cochlear nuclei have demonstrated auditory neuronal responses as early as 19 to 20 days of embryonic development in ducks (Konishi, 1973). Recently, optical recordings of auditory nerves of the Nuleus magnocellularis, Nuleus angularis, and ipsi- and contralateral Nucleus laminaris, have identified functional auditory circuits as early as embryonic day 7 in the domestic chick (Momose-Sato, Glover, \& Sato, 2006).

Visual system development in precocial birds also begins early in the prenatal period. One early indication of the onset of visual system development can be observed at approximately $30 \mathrm{hr}$ of incubation in the chicken when the prosencephalic neural tube begins to bulge out as it forms into the optic vesicles (LaVail \& Cowan, 1971). However, the functionality of the visual system involves the axonal production of retinal ganglion cells (RCG). Since all visual information reaches the chick brain through the axons of the RGC, and since these cells are the earliest to develop, their production is the first sign of development of a functional avian visual system (e.g., Kahn, 1973; 1974; Horder \& Mashkas, 1982). In addition, neurophysiologic evidence suggests that the retinotectal system of the chick embryo is functional between embryonic days 11-18 (Mathers \& Ostrach, 1979; McGraw \& McLaughlin, 1980). Given the evidence of early development and functionality of the auditory and visual system in precocial avain embryos, it is likely that multisensory interactions are possible during prenatal avian development. 
Although much is known about the neural mechanisms underlying attention and orientation to multisensory events during postnatal development, little is known as to whether selective attention and orientation can be modulated during prenatal development. For example, the primary role of the SC is to facilitate attention and orientation behaviors in space. This makes a difficult translation to prenatal attention and orientation because embryos and fetuses are bound within the confines of the egg or uterus. However, selective attention can be conceptualized in two distinct forms, endogenous, in which the organism voluntarily shifts its attention, or exogenous attention, in which shifts of attention are involuntarily evoked by external sensory events (see Spence \& McDonald, 2004; Vroomen \& de Gelder, 2004). Indeed work in the human adult literature on spatial orienting suggests that exogenous (covert) shifts of attention can take place prior to endogenous (overt) shifts of attention (e.g., Klien, Kingstone, \& Pontefract, 1992; Rafal, Henik, \& Smith, 1991; Rizzolatti, Riggio, Dascola, \& Umita, 1987; Shepperd, Findlay \& Hockey, 1986). Interestingly, the same neurophysiologic substrates, including the SC, mediate exogenous shifts of attention (Desimone, Wessinger, Thomas, \& Schneider, 1992; Robinson \& Kertzman, 1995; Stein, Wallace, \& Meredith, 1995; Thompson \& Masterton, 1978). Thus, it is likely that the neural enhancement effects caused by temporally synchronous audio-visual stimulation may also promote exogenous shifts of attention to integrated multisensory events without any overt behavioral responses during prenatal development being necessary.

However, many questions remain regarding the role that audio-visual temporal synchrony plays in prenatal perception. How does temporally synchronous information aid the infant or embryo to be able to detect various temporal properties of multisensory 
events? How does temporal synchrony guide and constrain selective attention, learning, and memory during late prenatal and early postnatal development? Does prenatal experience with multisensory temporal synchrony have enduring effects on postnatal perceptual processes? These questions are currently being explored in studies with both non-human animal and human infants. The following section will provide an overview of the principles of the Intersensory Redundancy Hypothesis and its role in providing a framework for explaining the early organization of intersensory perception. In addition, I will review some of the investigations with human infants on the effects of intersensory redundancy on attention and perception. A review of comparative studies on the effects of intersensory redundancy will follow, providing evidence that multisensory interactions are present in the prenatal period in precocial embryos.

The Effects of Intersensory Redundancy on Attention, Learning, and Memory

\section{during Early Development}

To address what features of multisensory stimulation are most salient to organisms during early development, Bahrick and Lickliter (2000) proposed an Intersensory Redundancy Hypothesis (IRH). Intersensory redundancy is defined as the temporally and spatially coordinated presentation of the same information across two or more sensory modalities. Amodal information such as tempo, rhythm, and intensity can be redundantly specified because they are available across more than one sensory modality (see Bahrick \& Pickens, 1994; Gibson \& Pick, 2000; Meltzoff \& Kuhl, 1994). Research has consistently demonstrated that young organisms are particularly adept at detecting amodal stimulus properties from redundantly specified multimodal events (e.g., Bahrick, 2002; Bahrick \& Lickliter, 2000; Lickliter \& Bahrick, 2001). 
The IRH provides several predictions for how young organisms attend to the amodal properties of multisensory events. Specifically, the IRH holds that, during early development, information that is available redundantly across two or more sensory modalities recruits infants' attention to amodal properties more readily than the same information made available via only one sensory modality. In addition, bimodallyspecified redundant information makes amodal properties stand out while attenuating the perception of modality specific properties. Thus, as an infant observes a bouncing ball, the amodal properties such as rhythm, temporal synchrony, and spatial colocation would become more salient to the young perceiver than modality specific properties such as the color (a modality specific property) of the ball.

The IRH also holds that information that is available via only one sensory modality (unimodal stimulation) or non-redundantly (asynchronous bimodal stimulation) facilitates the detection of modality specific properties and decreases the salience of amodal stimulus properties. Referring to the previous example, if a bouncing ball is presented without sound, the infant's detection of modality specific properties such as color would be facilitated at the expense of the detection of amodal properties. Intersensory redundancy thus guides and constrains early perceptual development by capturing (or attenuating) the infants' attention to various properties of multisensory events. It is important to emphasize, however, that the IRH pertains to early development. As infants gain experience in the world they acquire greater flexibility in their perceptual exploration of multimodal events. Thus, older infants are better able to switch their attention between amodal and modality specific properties during bouts of exploration of objects and events (Bahrick \& Lickliter, 2004). 
To test the tenets of the IRH, Bahrick and Lickliter (2000) conducted a series of experiments with 5-month-old infants to assess their ability to detect rhythm under bimodally redundant conditions. Specifically, infants were habituated to a video of a toy hammer tapping out a rhythm in one of three conditions: an audio-visual bimodal condition (intersensory redundancy), a unimodal visual or auditory condition, or a bimodal nonredundant (asynchrony) condition. The latter condition differed from the redundant bimodal condition in that the sights and sounds of the tapping hammer were not bimodally redundant; they were temporally asynchronous. In other words, intersensory redundancy was not available to the infants in this condition. This condition also served to control for the possibility that the bimodal condition was providing more stimulation than the unimodal conditions. During the habituation phase infants were exposed to the video of the tapping hammer until the total amount of looking time decreased to a criterion. Once infants reached the criterion, they were considered to be habituated to the stimulus and were subsequently presented with test trials of the toy hammer tapping out a different rhythm. Increased looking time to the novel stimulus was interpreted as the detection of a change in rhythm.

Results revealed that infants habituated to the bimodally redundant presentation of the tapping hammer detected a change in rhythm. However, in the condition under which infants were habituated to unimodal auditory or visual presentations of a tapping hammer, no evidence of visual recovery to a change in rhythm was found. The bimodal nonredundant (asynchrony) condition also revealed that infants did not visually recover to the change in rhythm. This study provided initial support for the IRH in that infants' ability to detect a change in rhythm (i.e., an amodal property) was facilitated by 
intersensory redundancy and was attenuated (or disrupted) when the stimulation was presented unimodally or asynchrnously. Subsequent studies using the same habituation paradigm with younger infants provide additional support for the IRH. Specifically, 3month-old infants' were found to discriminate a change in the tempo of a tapping toy hammer when habituated to bimodally redundant and temporally synchronous audiovisual presentation, but not unimodal presentations (Bahrick, Flom, \& Lickliter, 2002). An additional developmental prediction of the IRH holds that an infant's attentional skills will change with age and experience. Bahrick, Lickliter, and Flom (2006) provided evidence in support of this view. Specifically, 3, 5, and 8-month-old infants were assessed for their ability to detect a change in the orientation (up vs. down) of moving objects under either unimodal and/or bimodal conditions. Orientation is a nonredundantly specified property because it can be detected via the visual, but not the auditory modality. Infants from all 3 age groups were habituated to either a unimodal or bimodal redundant film of a tapping toy hammer in one of the two orientations, up or down. Subsequent test trials consisted of the same hammer tapping in a different orientation.

Results revealed that the 3,5, and 8-month-old infants showed significant visual recovery to a change in orientation under unimodal conditions. However, only 8-monthold infants were able to detect a change in orientation under both unimodal and bimodally redundant conditions. These results support the unimodal prediction of the IRH, that the detection of nonredundantly specified properties is facilitated when stimulation is made available unimodally. In addition, this study also provides evidence for the developmental prediction of the IRH. That is, as infants acquired more experience with 
objects and events, their perceptual exploration became more flexible, allowing the older infants to detect nonredundantly specified properties under either unimodal or bimodal conditions.

Comparative research has provided evidence that converges with the findings from human infants on the predictions of the IRH (Lickliter, Bahrick, \& Honeycutt, 2002, 2004). Specifically, research with bobwhite quail embryos has demonstrated that they are remarkably sensitive to intersensory redundancy. Lickliter, Bahrick, and Honeycutt (2002) assessed the effects of audio-visual intersensory redundancy on prenatal learning in bobwhite quail embryos. This study consisted of three experiments. In the three experimental groups, bobwhite embryos received exposure for either $10 \mathrm{~min} / \mathrm{hr}$ over the $24 \mathrm{hr}$ prior to hatching (240 min total exposure time), $10 \mathrm{~min} / \mathrm{hr}$ over the $12 \mathrm{hr}$ prior to hatching (120 $\mathrm{min}$ ), or $10 \mathrm{~min} / \mathrm{hr}$ over the $6 \mathrm{hr}$ prior to hatching (60 min). In Experiment 1, embryos received unimodal exposure to an individual variant of a bobwhite maternal call. In Experiment 2, prenatal exposure consisted of a concurrent presentation of a patterned light that was temporally asynchronous to the maternal call. In Experiment 3, the presentation of the patterned light was temporally synchronous with the notes of the call, thus providing intersensory redundancy to the embryos. All subjects were subsequently given a 5 minute simultaneous choice test between the familiar bobwhite maternal call and a nonfamiliar bobwhite maternal call at $24 \mathrm{hr}$ following hatching.

The results of this study demonstrated that embryos exposed to $240 \mathrm{~min}, 120 \mathrm{~min}$, and 60 min of intersensory redundancy (Experiment 3) demonstrated enhanced prenatal perceptual learning of the familiar maternal call as evidenced by significant preferences during postnatal tests. In contrast, only the embryos that received 240 min of prenatal 
exposure to the unimodal auditory call (Experiment 1) showed a significant preference for the familiar call during postnatal tests. Bobwhite quail neonates that received prenatal exposure to the asynchronous audio-visual call did not demonstrate a preference for the familiar call for any of the three exposure durations (i.e., $240 \mathrm{~min}, 120 \mathrm{~min}, \& 60 \mathrm{~min}$ ). Thus, when the maternal call was redundantly specified, bobwhite quail embryos required $1 / 4$ of the exposure amount to learn the call. These comparative findings highlight the functional role that redundant bimodal stimulation plays in guiding attention and perceptual learning during early development as compared to unimodal, or asynchronous stimulation.

In addition to intersensory redundancy facilitating prenatal perceptual learning, intersensory redundancy can also enhance memory during the perinatal period. Using the same procedures as in Lickliter, Bahrick, and Honeycutt (2002), a series of experiments (Lickliter, Bahrick, \& Honeycutt, 2004) were conducted in which embryos received either unimodal auditory exposure to a bobwhite maternal call for $10 \mathrm{~min} / \mathrm{hr}$ for $24 \mathrm{hr}$ prior to hatching (240 min total exposure time), a temporally synchronous (or redundant) audio-visual maternal call for the same amount of exposure time, or no supplemental prenatal stimulation. Subjects from all the groups were subsequently individually tested at $48 \mathrm{hr}$ and $72 \mathrm{hr}$ following prenatal stimulation offset in a simultaneous choice test that assessed their preference for either the familiar maternal call or an unfamiliar maternal call. Results demonstrated that embryos that received prenatal exposure to the unimodal maternal call and embryos that received no supplemental stimulation did not show a significant preference for either the familiar maternal call or the nonfamiliar call at both the $48 \mathrm{hr}$ and $72 \mathrm{hr}$ tests. In contrast, embryos that received exposure to the bimodally 
redundant call showed significant preferences for the familiar maternal call at the $48 \mathrm{hr}$ test, but not at the $72 \mathrm{hr}$ test, demonstrating that intersensory redundancy can double the amount of retention time as compared with exposure to the same call unimodally.

Just how effective can intersensory redundancy be at enhancing memory during prenatal development? An additional experiment to further investigate the effects of prenatal experience with intersensory redundancy on memory was conducted. The authors were specifically interested in whether a brief postnatal re-familiarization to the auditory call would benefit those embryos that received intersensory redundancy. The idea being that re-familiarization to information that is no longer in memory (in this case the maternal call) results in a phenomenon called "memory savings," characterized by a re-learning of the already forgotten information (Alder, Wilk, \& Rovee-Collier, 2000; Cornell, 1979; Monk, Gunderson, Grant, \& Mechling, 1996; Nelson, 1985). As in the previous experiments, embryos were exposed either to a temporally synchronized redundant call, a unimodal auditory call, or no supplemental stimulation. Following hatching, chicks from each of the three experimental conditions were provided with one 10-min session at $48 \mathrm{hr}$. A separate group of chicks from the redundant group also received a re-familiarization exposure at $72 \mathrm{hr}$ after hatching. As predicted, the embryos exposed to intersensory redundancy preferred the familiar call at $72 \mathrm{hr}$ and $96 \mathrm{hr}$ after hatching. Embryos from the unimodal and control group did not show evidence of memory savings. Thus, chicks that learned the call containing intersensory redundancy were able to remember the call four times longer than those embryos that were exposed to the call unimodally. 
Intersensory redundancy can also play a role in guiding selective attention to amodal stimulus properties of unimodal stimulation. For example, Lickliter, Bahrick, and Markham (2006) exposed a group of bobwhite quail embryos with $6 \mathrm{hr}$ of redundant bimodal exposure to a maternal call followed by $18 \mathrm{hr}$ of unimodal exposure to the same maternal call in the $24 \mathrm{hr}$ prior to hatching. Another group received the reverse order of stimulus presentation such that they first received exposure to $18 \mathrm{hr}$ of a unimodal maternal call followed by $6 \mathrm{hr}$ of the same call presented bimodally redundant. The former group (6hr bimodally redundant call $\rightarrow 18 \mathrm{hr}$ unimodal call) demonstrated a significant preference to the familiar call during postnatal tests. In contrast, embryos that received the reverse order of stimulation ( $18 \mathrm{hr}$ unimodal call $\rightarrow 6 \mathrm{hr}$ bimodally redundant call) did not demonstrate a preference for the familiar call during postnatal tests. These findings suggest that initial exposure to a stimulus event containing intersensory redundancy, in which the amodal properties of the event are salient, can educate a young organism's selective attention to the same amodal stimulus properties when the stimulus event is presented unimodally. Taken together these results indicate that intersensory redundancy plays a key role in early perceptual learning, even during prenatal development.

\section{Current Study}

Linking the effects of prenatal experience to postnatal intersensory development is particularly challenging because the environment of a fetus or embryo is quite different from that of a human or animal infant. In the prenatal environment, for example, sensory stimulation is attenuated. Visual stimulation may be in the form of a subtle change in light intensity and the acoustical properties of sound may be muffled and unclear. 
Embryonic or fetal movement is also constrained, further limiting the opportunity for exploration of multisensory events. Despite these differences, the prenatal environment shares a similar characteristic with the postnatal environment in that it contains sensory stimulation that is temporally synchronized. For example, fetuses receive ongoing exposure to temporally synchronized vibrations and sounds of their mother's respiration, heartbeat, and vocalizations. There is evidence demonstrating that fetuses are indeed sensitive to these internal and external sounds and vibrations (see Fifer \& Moon, 1995 for a review). Given that fetuses and embryos are exposed to some of the temporal amodal properties they will experience postnatally, it is plausible that prenatal experience with temporal synchrony serves as an initial basis for the development of attentional and perceptual processes during postnatal development. Little is known, however, as to exactly how prenatal sensory experience with temporal amodal properties contributes to early intersensory development.

An area that has received limited investigation is the range of an embryo's ability to attend to various types of temporal configurations of bimodal stimulation. What features of bimodal redundancy play are critical to "grab" the embryo's attention and facilitate perceptual learning? It has been established that an important feature of intersensory redundancy that facilitates prenatal perceptual learning is the temporal synchrony of bimodal stimulation. Further, previous comparative studies with bobwhite quail chicks have demonstrated that concurrent but asynchronous prenatal exposure to bimodal stimulation disrupts prenatal learning (Honeycutt \& Lickliter, 2001; Lickliter \& Hellewell, 1992). This asynchronous stimulation has been shown to elevate the embryos' physiological arousal, likely contributing to a shift in attentional processes (Reynolds \& 
Lickliter, 2002). In contrast, temporally synchronized information has been shown to enhance learning and memory in avian embryos and hatchlings (e.g., Hultsch, Scheuss, \& Todt, 1999; Lickliter, Bahrick, \& Honeycutt 2002, 2004).

This current study was designed to determine the role that temporal synchrony plays in facilitating prenatal attention and learning. Are avian embryos sensitive to different forms of redundancy that do not involve the temporal overlap (temporal synchrony) of two sensory modalities? Or is temporal synchrony a critical feature for the facilitation of attention and learning? There is evidence in the comparative developmental literature that infant animals differ in how simultaneous vs. sequential events are learned. For example, infant rats 8 to 16 days of age outperform older animals at learning an association between pre-conditioned and neutral olfactory stimuli, but only with simultaneous presentations of the odors; sequential presentation eliminates or reverses the infant rats' advantage (Chen, Lariviere, Heyser, Spear, \& Spear, 1991). Even at $3 \mathrm{hr}$ of age newborn rats exhibit robust sensory preconditioning and second-order conditioning (Cheslock, Varlinskaya, High, \& Spear, 2003). These results suggest that there is weak differentiation among synchronous stimuli in very young organisms that may promote something similar to perceptual configuration or "unitization" of stimuli presented simultaneously.

Although previous work with bobwhite quail embryos has suggested that amodal invariants are responsible for recruiting attention and facilitating perceptual learning (e.g., Lickliter, Bahrick, \& Honeycutt, 2002, 2004), it is not clear whether this applies to all amodal invariants that were present in equivalent stimulation provided or whether it was simply the temporal synchrony in the stimulus event. In this light, Lewkowicz and 
Kraebel (2004) have argued that, "The real issue is whether infants can detect the specific temporal pattern as an independent perceptual feature regardless of modality (i.e., true amodal invariant)..." To date, however, the evidence is not clear on this possibility, and convincing proof will require a demonstration that infants can perceive intersensory equivalence of various amodal invariants in the absence of intersensory temporal synchrony" (p. 671). From a methodological standpoint, it is difficult to present young organisms with redundant audio-visual stimulation without temporal synchrony present. To address this issue one would have to separate the concurrent and redundant bimodal information and present it in a sequential manner in an attempt to preserve the temporally equivalent nature of the bimodal event (but not have temporal synchrony available in the stimuli). For example, if amodal temporal properties are made available to both visual and auditory sensory modalities, then the same amodal properties can be presented sequentially alternating between the visual and auditory modalities, but without the stimulation being temporally synchronized. From here on, I will refer to this form of stimulation as Bimodal Sequential Temporal Equivalence (BSTE).

To date no studies have explored whether BSTE is sufficient for effective facilitation of prenatal perceptual learning and addressing this issue could shed light on the importance of temporal synchrony in the early organization of intersensory perception. Although it is possible to detect amodal invariants unimodally during prenatal development, redundancy (i.e., temporally synchronous bimodal stimulation) is thought to make amodal properties particularly salient to quail embryos (Lickliter, Bahrick, \& Honeycutt, 2002). One focus of the current study explores whether quail embryos find BSTE a salient form of bimodal stimulation and whether the temporal 
overlap (e.g., temporal synchrony) of audio-visual stimulation is not needed for facilitating prenatal perceptual learning.

A second focus of this study was to begin to describe the critical components that make bimodal stimulation salient during prenatal development and to delineate what features of bimodal redundancy promote selective attention and facilitate perceptual learning during prenatal development. In particular, I explored the critical amount and the timing of occurrence of temporal synchrony that facilitates the rate of learning of species-specific stimulation during prenatal development. What constitutes "effective" synchrony to the embryo, what aspects of bimodal synchrony are "attention grabbing" during early development, and how do they foster the rapid learning of multimodal information?

Thus, this study examined the following three related hypotheses:

(1) The availability of temporal synchrony is a critical feature to facilitate prenatal perceptual learning with respect to unimodal stimulation. As previously discussed, temporal synchrony is the basis for increased neurophysiological and behavioral responsiveness to bimodal stimulation. Thus, redundant stimulation without temporal synchrony will not facilitate prenatal perceptual learning with respect to unimodal stimulation.

(2) A single temporally synchronous note is sufficient to facilitate prenatal perceptual learning with respect to unimodal stimulation. This is based on previous evidence demonstrating that bobwhite quail embryos benefit from overlapping and synchronized audio-visual stimulation. Thus, prenatal perceptual learning is facilitated 
with respect to unimodal stimulation because of the availability of temporal synchrony, not the overall amount of sensory information available.

(3) In situations where embryos are exposed to a single temporally synchronous note, facilitated learning with respect to unimodal stimulation will be optimal when the temporally synchronous note occurs at the onset of the stimulation bout. Previous

evidence has demonstrated that initial exposure to a redundant audio-visual maternal call educates the embryo's selective attention to amodal stimulus properties of the auditory call in subsequent unimodal presentations (Lickliter, Bahrick, \& Markham, 2006). Similarly, brief audio-visual synchrony made available at the onset of a stimulus event should direct the embryos' attention to the amodal properties present in a unimodal stimulus event, thus facilitating prenatal perceptual learning with respect to unimodal stimulation.

\section{General Method}

Certain features of the experimental design were common to all experiments. These features are described first before describing the particular details of each experiment.

Subjects

Subjects were incubator reared bobwhite quail chicks (Colinus virginianus). Fertilized unincubated eggs were received weekly from a commercial supplier and set in a BSS-160 Grumbach Incubator maintained at $75-80 \%$ relative humidity and $37.5^{\circ} \mathrm{C}$. Embryonic age was calculated on the basis of the first day of incubation as Day 0, second day of incubation as Day 1, and so on. To control for possible variations in developmental age, only those birds that hatched on Day 23 were used as subjects. 
Embryos for each condition were drawn from two or more different batches of eggs to control for possible between-batch variation in behavior. Following hatching, groups of 15-20 chicks were reared in large plastic tubs in a Nuaire Model NU-605-500 Animal Isolator (Plymouth, MN), which provided continuous filtered air. Chicks had continuous access to food and water. Ambient air temperature was maintained at approximately $30^{\circ} \mathrm{C}$.

Apparatus

Approximately 24 hrs prior to hatching, embryos were transferred to a sound attenuated stimulation room and placed in a Model 1602N Hova-Bator portable hatcher, maintained at approximately $37.5^{\circ} \mathrm{C}$ and $80 \%$ relative humidity. This hatcher allowed embryos to receive audio-visual stimulation via a transparent plastic window located directly above the embryos. Audio-visual stimulus presentations (described below) were delivered via a custom designed software program running a flat screen video monitor located $22 \mathrm{~cm}$ directly above the portable hatcher window and a speaker placed on top of a small hole located on the top of the hatcher, immediately adjacent to the window.

Postnatal behavioral tests took place in an arena $130 \mathrm{~cm}$ in diameter, surrounded by a wall $60 \mathrm{~cm}$ in height. The arena surface was painted flat black and the walls of the testing arena were insulated with a special layer of foam to attenuate reverberation. The arena walls were covered with an opaque black curtain. A video camera mounted directly above the arena allowed for remote observation and data collection. Two semicircular approach areas, each comprising approximately $5 \%$ of the total area of the testing arena and directly opposite to one another, were demarcated on a remote video monitor (these were marked on the testing arena's substrate, Figure 1). The two approach areas 
contained a 4 inch speaker mounted to the arena wall and hidden behind the black curtain (to allow for auditory stimulation). Both speakers were powered by separate RCA SA155 integrated stereo amplifiers and the auditory stimuli were played by two Sony CDPXE370 compact disk players. Ambient room temperature was maintained between $29^{\circ}$ $32^{\circ} \mathrm{C}$

\section{Figure 1}

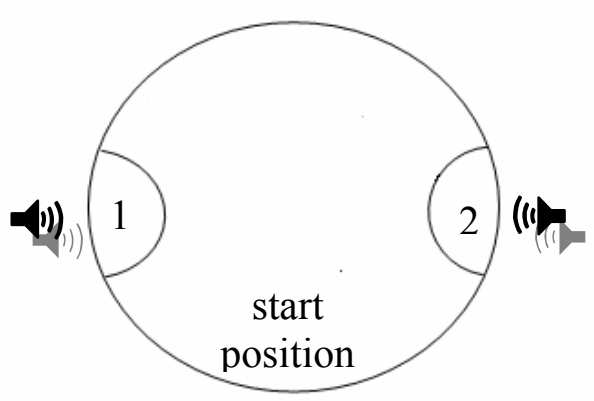

Top View of the Behavioral Testing Arena.

Approach areas " 1 " and " 2 " contained either the familiar call or non-familiar call. Familiar call location was counterbalanced during testing.

\section{Procedure}

Approximately 24-36 hr prior to hatching, the bobwhite quail embryo moves its head into the airspace located at the large end of the egg producing a visible indentation, or pip, on the surface of the egg shell. Approximately $24 \mathrm{hr}$ prior to hatching 25 "pipped" eggs were relocated to a portable hatcher in a light and sound attenuated prenatal stimulation room. Various sensory stimulation regimes were presented for a total duration of $10 \mathrm{~min} / \mathrm{hr}$ during the last $18 \mathrm{hr}, 12 \mathrm{hr}$, or $6 \mathrm{hr}$ prior to hatching. The auditory stimuli used were two individual variants of a species-typical bobwhite maternal assembly call (Call A and Call B). Both maternal calls were recorded in the field 
(Heaton, Miller, \& Goodwin, 1978) and share similar phrasing, repetition rates, and frequency modulation. They vary primarily in minor peaks of dominant frequency and the temporal microstructure of rhythm and duration (see Figure 2).

Figure 2

Spectrograms of the Bobwhite Maternal Calls (A and B).

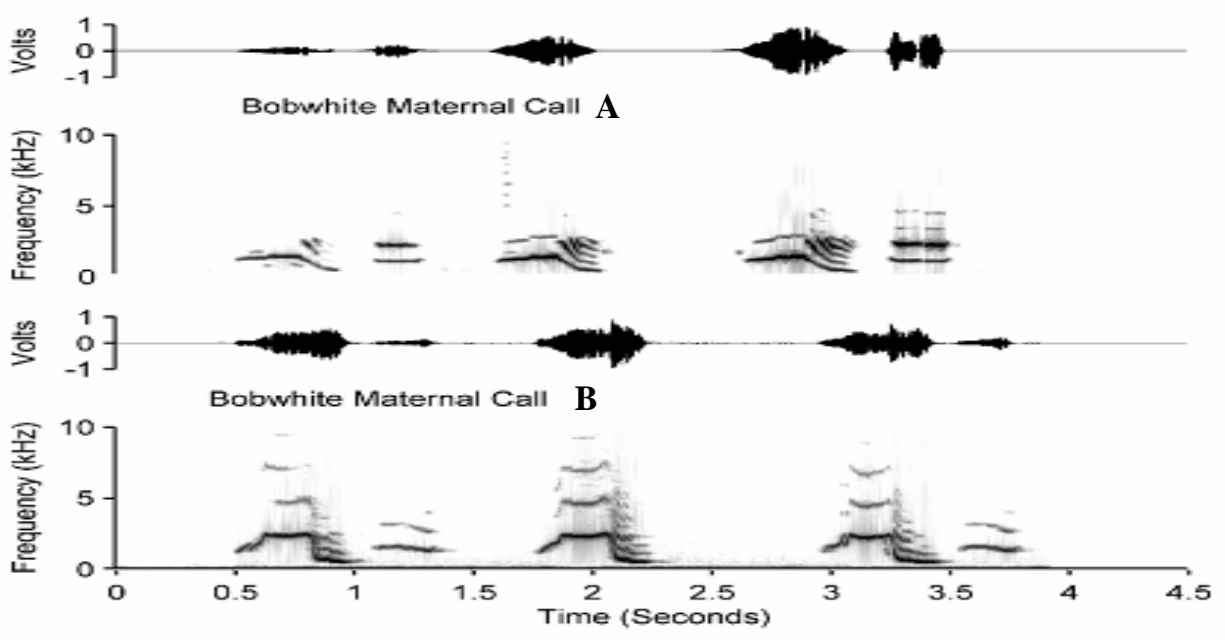

For each condition, $50 \%$ of subjects received prenatal exposure to Call A and $50 \%$ of subjects received prenatal exposure to Call B. These maternal calls consist of a burst of five notes with a complex rhythmic pattern. The duration of the burst is $3 \mathrm{sec}$ (the rate of notes average 1.7 notes per second) and is followed by an inter-burst interval of 2 second. The notes of the call vary in duration, intensity, and fundamental frequency. Various audio-visual configurations of the maternal call were achieved by presenting the pulsed light either in synchrony or in sequential order with certain notes of the maternal call. A customized version of the computer software Javascript was used to create the stimulation regimes. 


\section{Testing}

Testing was conducted $24 \mathrm{hr}$ following prenatal stimulation completion and consisted of a 5 min (300s) simultaneous-choice test between the unimodal familiar bobwhite call, used during prenatal stimulus exposure (Call A or Call B) and a novel maternal call (call A or call B). Thus, chicks could respond to call A or call B during testing. The sound intensity of each call was adjusted to peak at $65 \mathrm{~dB}$, measured from the start position where each chick was introduced into the arena. The locations of the calls presented during testing were counterbalanced across individual trials to prevent a possible side bias from affecting results. Each chick was tested only once. Chicks were scored on both their latency of approach and the duration of time they spent in each of the approach areas by an observer blind to the experimental condition. Latency was defined as the amount of time (in seconds) that elapsed from the onset of the trial until the chick entered an approach area. Duration was defined as the cumulative amount of time (in seconds) the chick remained in an approach area.

Any chick that did not enter any approach area received a score of 300 seconds for latency (i.e., the length of the trial) and 0 seconds for duration and was considered a non-responder. These chicks were excluded from subsequent analyses. A preference for a given call was scored if a chick stayed in an approach area for at least twice the time spent in the opposing approach area. No preference for a stimulus was scored if a chick approached the two areas during a trial but did not spend at least twice as much time in one approach area as the other. These measures of preference constituted the primary dependent variable. A Visual Basic computer program allowed for semi-automated collection of latency and duration of response to the test stimuli. 


\section{Data Analysis}

Previous perceptual studies with bobwhite quail have used nonparametric tests as the primary form of analysis. For this study, parametric tests were used as primary analyses because it allowed for the evaluation of between-group differences.

Nonparametric tests were used as secondary analyses. However, due to the high variability of the interval data, it was expected that the results of the parametric and nonparametric tests would not always correspond. Based on these circumstances, a criterion was established to maximize the utility of both parametric and nonparametric tests. Specifically, for a group to be considered as having their prenatal perceptual learning facilitated, at least one primary (parametric) and one secondary (nonparametric) test had to result in statistical significance. Groups that did not meet this criterion were interpreted as showing non-significant preference, hence prenatal perceptual learning was not facilitated.

For the primary analyses, the data of interest were measures of duration (in seconds) for the auditory stimuli presented during the test trials. Several analyses were performed on this interval data. First, a proportion of total duration time (PTDT) was calculated from the time chicks spent in the approach area containing the familiar maternal call relative to the total duration time spent in both familiar and novel approach areas. A proportion of .50 reflects chance responding, whereas a proportion greater than .50 reflects a majority of time spent in the approach area containing the familiar call. Proportions less than .50 reflect a majority of time spent in the approach area containing the novel call (see Bahrick 2002; Bahrick \& Lickliter, 2004 for examples). One Sample $t$ tests were used to evaluate whether the PTDT spent in the approach area containing the familiar call was 
significantly greater than chance. In addition, between groups comparisons of PTDT to the familiar call were evaluated with a One-Way Analysis of Variance (ANOVA) and a multiple comparison procedure (e.g., Tukey's HSD test).

Secondary nonparametric analyses were performed on the duration of time spent in each approach area by subjects in a group by the Wilcoxon Matched-Pairs SignedRanks Test. This test evaluated whether the duration scores for the familiar stimulus were longer from that of the novel stimulus within a condition. In addition, an individual preference score (used in a number of prior studies of perceptual discrimination in bobwhite quail, see Lickliter \& Hellewell, 1992; Lickliter \& Lewkowicz, 1995 for examples), was assigned to any chick that stayed in one area for more than twice as long as the other area. These preferences were evaluated by the Chi-Square Goodness-of-Fit Test to determine if subjects in a condition showed a significant preference for the familiar call vs. novel call presented in the simultaneous-choice test. Significance levels of $p<.05$ (two-tailed) were used to evaluate all results. Latency of initial approach to the familiar and novel calls was also recorded for each subject. However, these data turned out to be highly variable across subjects and will not be discussed further in the individual experiments.

Experiment 1: Temporal Synchrony as a Critical Feature for the Facilitation of Prenatal Perceptual Learning

Recently, temporal synchrony has been proposed as the basis for the salience of redundant multisensory stimulation during early development (Lewkowicz \& Kraebel, 2004; Prince \& Hollich, 2005). In addition, researchers exploring computational models of multisensory perception have demonstrated that temporal synchrony facilitates the 
initial detection of multisensory events (Fitzpatrik, Aresenio, \& Torres-Jara, 2006).

Previous research has also demonstrated that temporally synchronous audio-visual stimulation (intersensory redundancy) enhances the rate of prenatal perceptual learning and memory in bobwhite quail (Lickliter, Bahrick, \& Honeycut, 2002, 2004). This experiment extends the existing research on prenatal perceptual learning and explores whether facilitated prenatal perceptual learning can be achieved on the basis of temporal equivalence without the presence of temporal synchrony in bimodally redundant stimulation.

In order to unpack temporal synchrony from bimodally redundant stimulation, a group of embryos were exposed to Bimodal Sequential Temporal Equivalence (BSTE). BSTE is a form of bimodal stimulation that does not contain the property of temporal synchrony however the amodal properties are sequentially and temporally equivalent to those in bimodal synchronous stimulation. In other words, the same temporal amodal properties present in the visual modality are available in the auditory modality and occur in a sequentially alternating pattern (Figure 3c). This allowed for assessing the role of temporal equivalence in the facilitation of prenatal perceptual learning independent of temporal synchrony. It was predicted that temporal synchrony would be a critical component in the facilitation of prenatal perceptual learning. Thus, embryos that received exposure to BSTE should not demonstrate enhanced prenatal perceptual learning. In contrast, as reported in earlier studies (Lickliter, Bahrick, \& Honeycutt, 2002, 2004), embryos exposed to audio-visual stimulation containing temporal synchrony should show enhanced prenatal perceptual learning. 
What constitutes facilitated prenatal perceptual learning? Previous research has demonstrated that quail embryos exposed to a (unimodal) maternal call require $240 \mathrm{~min}$ of total exposure time in order for prenatal perceptual learning to occur, whereas $120 \mathrm{~min}$ of unimodal exposure is not sufficient to foster prenatal perceptual learning. In contrast, when embryos receive exposure to a temporally synchronous audio-visual maternal call (intersensory redundancy), prenatal perceptual learning was facilitated with respect to the amount needed for prenatal perceptual learning of a unimodal auditory call (Lickliter, Bahrick, \& Honeycutt, 2002). That is, embryos exposed to 120 min of a temporally synchronized audio-visual maternal call demonstrated a significant preference for the familiar call in postnatal tests. In fact, just 60 min of prenatal exposure to a temporally synchronized maternal call was sufficient for embryos to learn the familiarized call whereas more unimodal was required for embryos to learn the same call. Thus, embryos can learn a maternal call four times faster when exposed to a temporally synchronous audio-visual version of that maternal call than when exposed to the same unimodal auditory maternal call as compared with unimodal stimulation. For purposes of this study, prenatal perceptual learning occurring with just 120 min or 60 min of prenatal exposure time constitutes facilitated prenatal perceptual learning.

\section{Method}

Bobwhite quail embryos were assigned to one of four groups: (1) a Unimodal group ( $\mathrm{N}=57)$ which received exposure to an individual variant of the bobwhite maternal call (Figure 3a). This group served to explore the minimum amount of unimodal stimulation needed for prenatal perceptual learning and was divided into two subgroups of different exposure amounts. Group A (N=29) received exposure every $10 \mathrm{~min} / \mathrm{hr}$ for 
$24 \mathrm{hr}$ prior to hatching (240 min of total stimulation). Group $\mathrm{B}(\mathrm{N}=28)$ received exposure every $10 \mathrm{~min} / \mathrm{hr}$ for $12 \mathrm{hr}$ prior to hatching (120 min of total stimulation), (2) a $100 \%$ Synchrony group $(\mathrm{N}=60)$ received exposure to a maternal call such that the temporal patterning of the auditory call notes was recreated in a pulsed light. Thus, the amodal properties of rhythm, rate, and duration were redundant and temporally synchronized in both the auditory and visual modalities (Figure 3b). As with the unimodal group, this group was used to explore the minimum amount of bimodal synchronous stimulation needed for prenatal perceptual learning and was also divided into two subgroups. Group A $(\mathrm{N}=30)$ received exposure every $10 \mathrm{~min} / \mathrm{hr}$ for $12 \mathrm{hr}(120$ min of total stimulation) and Group $\mathrm{B}(\mathrm{N}=30)$ received exposure every $10 \mathrm{~min} / \mathrm{hr}$ for $6 \mathrm{hr}$ (60 min of total stimulation), (3) a Bimodal Sequential Temporal Equivalent (BSTE) group $(\mathrm{N}=30)$ received exposure to an individual variant of the bobwhite maternal call for 3 seconds immediately followed by 3 seconds of a pulsing light containing the same temporal properties of the call, followed by another 3 seconds of the maternal call, and so on. The inter-stimulus-interval between the auditory and visual bursts was 3.4 seconds (Figure 3c). To determine whether BSTE could facilitate prenatal perceptual learning, this group received $10 \mathrm{~min} / \mathrm{hr}$ for $12 \mathrm{hr}$ (120 min of total stimulation) of exposure prior to hatching, (4) and a Control group (N=31), which did not receive any supplemental prenatal stimulation.

Testing for all experimental groups was conducted at $24 \mathrm{hr}$ following hatching in a 5 min simultaneous-choice test between the familiarized unimodal auditory maternal call and a novel unimodal auditory variant of the bobwhite maternal call. It is important to emphasize that the maternal call variants presented at testing did not contain the visual 
component. Because neither of the call variants was familiar to the control group chicks, the 5 min simultaneous-choice test was given to each chick to determine whether there was a naïve preference for either of the two maternal call variants (call $\mathrm{A}$ and call $\mathrm{B}$ ). To counterbalance calls, half of the subjects from the control group received the 5 min test with call $\mathrm{A}$ in area 1 and call $\mathrm{B}$ in area 2, and half the subjects with the calls in the reverse order. After testing, each chick was assigned one of three preference scores: (1) a preference for Area 1, (2) a preference for Area 2, or (3) no preference. A Chi square test was applied to the preference scores to evaluate whether there was a naïve preference for either maternal call variant.

Figure 3

Schematic Representation of the Various Audio-Visual Configurations of the Maternal Call used in Experiment 1. The letters $\mathrm{A}$ and $\mathrm{V}$ represent the auditory and visual modalities, respectively. The numbers represent each note in the 5-note call burst. The duration of each call burst is 3 seconds and the duration of the inter-burst-interval is 2.4 seconds.

(a) Unimodal

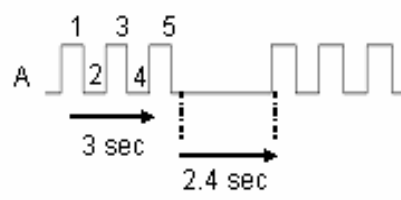

(b) $100 \%$ synchrony

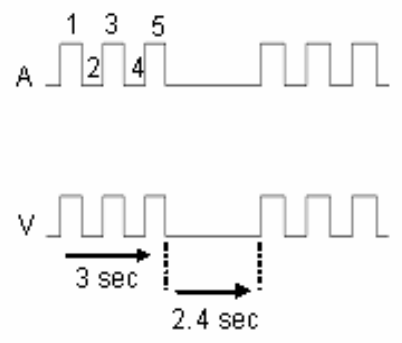

(c) BSTE

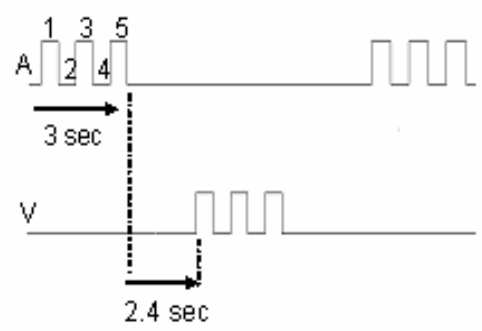

Results and Discussion

The results for the experimental groups are illustrated in Figure 4. One-sample ttests were performed on the proportion of total duration time (PTDT) spent in the approach area with the familiar call against the chance value of .50 on all experimental groups. Results revealed that embryos receiving 240 min of a Unimodal maternal call showed a 
greater PTDT to the familiar call at testing $t(28)=3.130, \mathrm{p}=.004$. Likewise, embryos from the $100 \%$ synchrony $(120 \mathrm{~min})$ and $100 \%$ synchrony $(60 \mathrm{~min})$ groups showed a greater PTDT spent in the approach area with the familiar call at testing, $t(29)=6.730, \mathrm{p}$ $=.000$ and $t(29)=2.906, \mathrm{p}=.007$, respectively $($ Table 1$)$.

A one-way analysis of variance compared the mean PTDT to the familiar call of all experimental groups. This test was found to be statistically significant at an alpha level of $.05, \mathrm{~F}(4,142)=2.842, \mathrm{p}=.026$. A Tukey HSD test indicated that the mean PTDT to the familiar call for the $100 \%$ Synchrony 120 min group $(\underline{\mathrm{M}}=.809, \mathrm{SD}=.251)$ was significantly greater than the mean PTDT for the Unimodal 120 min group $(\underline{\mathrm{M}}=.541, \mathrm{SD}$ $=.344)$. The mean PTDT for the Unimodal 240 min group $(\underline{\mathrm{M}}=.679, \mathrm{SD}=.323), 100 \%$ Synchrony 60 min group $(\underline{\mathrm{M}}=.656, \mathrm{SD}=.294)$ and the BSTE 120 min group $(\underline{\mathrm{M}}=.615$, $\mathrm{SD}=.368)$ did not differ significantly from the mean PTDT for the other groups.

Figure 4

Mean Proportion of Total Duration Time for the Familiar Call in Experiment 1

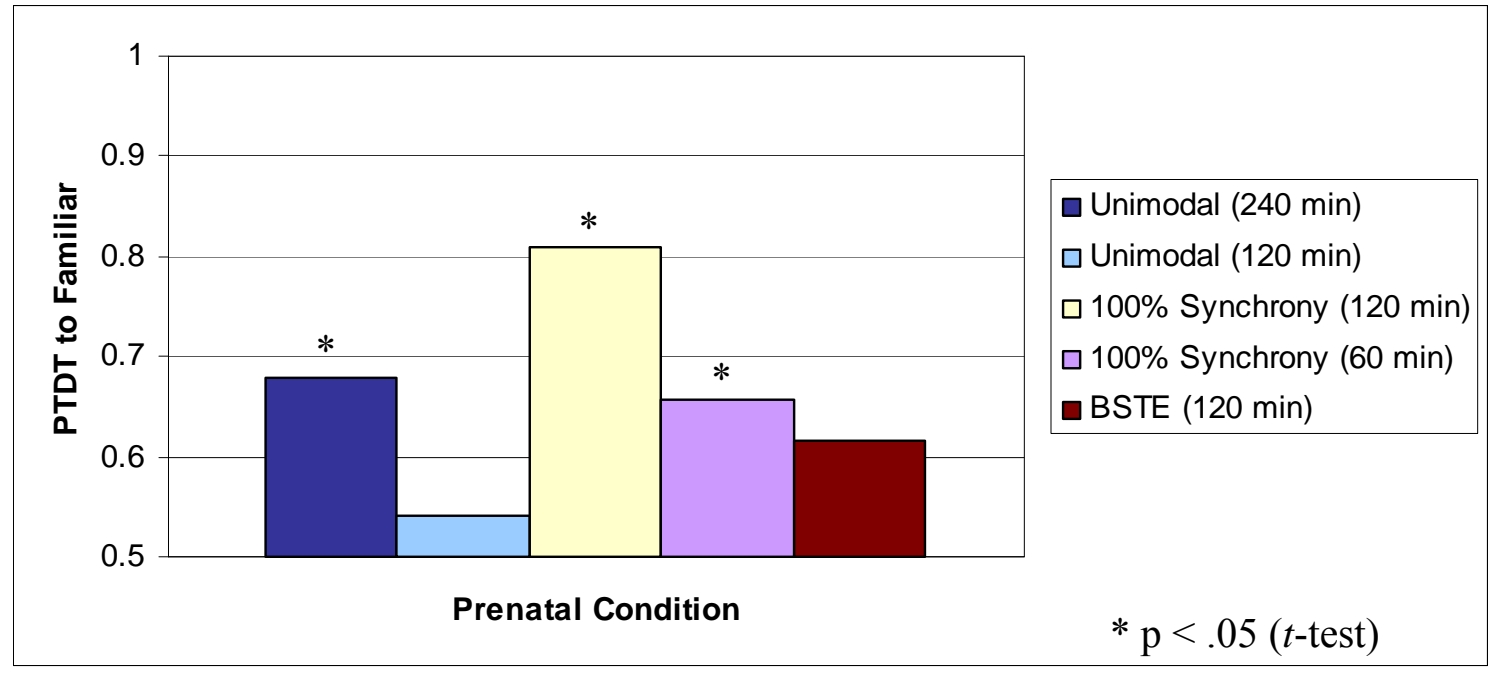


Table 1

Mean Proportions of Total Duration Time and Standard Deviations (in seconds) for the Familiar Call for Experiment 1

\begin{tabular}{lll}
\hline Unimodal $(240 \mathrm{~min})$ & $\mathrm{x}=.679$ & $(\mathrm{SD}=.307)^{*}$ \\
Unimodal $(120 \mathrm{~min})$ & $\mathrm{x}=.541$ & $(\mathrm{SD}=.344)$ \\
$100 \%$ Synchrony $(120 \mathrm{~min})$ & $\mathrm{x}=.809$ & $(\mathrm{SD}=.251)^{*}$ \\
$100 \%$ Synchrony $(60 \mathrm{~min})$ & $\mathrm{x}=.656$ & $(\mathrm{SD}=.295)^{*}$ \\
BSTE $(120 \mathrm{~min})$ & $\mathrm{x}=.615$ & $(\mathrm{SD}=.368)$ \\
\hline$* \mathrm{p}<.05(t$-test $)$ & &
\end{tabular}

A Wilcoxon signed-rank test was also applied to the duration measure within conditions and revealed that chicks prenatally exposed to the Unimodal (240 min) group had significantly longer durations to the approach area containing the familiar call, $\mathrm{z}=$ $2.368, \mathrm{p}=.018$. The $100 \%$ Synchrony (120 min) group also demonstrated significantly longer durations to the familiar call, $\mathrm{z}=-4.206, \mathrm{p}=.000$, as did the $100 \%$ Synchrony $(60$ $\min$ ) group, $\mathrm{z}=-2.561, \mathrm{p}=.010$ (Table 2). A Chi-square goodness-of-fit test was applied to the relationship between prenatal condition and number of preferences for the familiar call for each group and found to be statistically significant at an alpha level of .05 for the Unimodal $(240 \mathrm{~min})$ group, $\chi^{2}(2, N=29)=6.276 ; \mathrm{p}=.043$ and the $100 \%$ Synchrony $(120$ $\min$ ) group, $\chi^{2}(2, N=31)=25.80 ; p=.000$ (Figure 5). It is important to note that this analysis found the $100 \%$ Synchrony (60 $\mathrm{min})$ group statistically non-significant, $\chi^{2}(2, N=$ $30)=4.20 ; p=.122$. Observed preference scores are shown in Table 3a. A Chi-square test was also applied to the Control group however the preferences evaluated were between Area 1, Area 2, and No Preference. Results of the Chi-square test revealed that naïve 
chicks did not show a statistically significant preference for either maternal call variant at testing. Observed preference scores for the Control group are shown in Table $3 b$.

Table 2

Means and Standard Deviations of Duration Scores (in seconds) for Experiment 1

\begin{tabular}{|c|c|c|c|}
\hline \multirow[b]{2}{*}{ Prenatal Condition } & \multirow[b]{2}{*}{$\mathrm{N}$} & \multicolumn{2}{|c|}{ Duration } \\
\hline & & Familiar Call & Novel Call \\
\hline Unimodal (240 min) & 29 & $\begin{array}{c}27.87^{*} \\
(31.98)\end{array}$ & $\begin{array}{c}12.54 \\
(11.91)\end{array}$ \\
\hline Unimodal (120 min) & 28 & $\begin{array}{c}67.71 \\
(61.76)\end{array}$ & $\begin{array}{c}63.84 \\
(63.43)\end{array}$ \\
\hline $100 \%$ Synchrony (120 min) & 30 & $\begin{array}{l}102.46^{*} \\
(67.15)\end{array}$ & $\begin{array}{l}18.82 \\
(25.04)\end{array}$ \\
\hline $100 \%$ Synchrony $(60 \mathrm{~min})$ & 30 & $\begin{array}{c}72.53^{*} \\
(55.22)\end{array}$ & $\begin{array}{c}34.30 \\
(48.02)\end{array}$ \\
\hline BSTE (120 min) & 30 & $\begin{array}{c}47.97 \\
(56.11)\end{array}$ & $\begin{array}{c}37.13 \\
(41.48)\end{array}$ \\
\hline
\end{tabular}

Table 3a

Preference Scores for Conditions in Experiment 1

\begin{tabular}{lcccc}
\hline & & \multicolumn{3}{c}{ Preference Category } \\
\cline { 3 - 5 } Prenatal Condition & $\mathrm{N}$ & Familiar & Novel & No Preference \\
\hline Unimodal (240 min) & 29 & $* 16$ & 7 & 6 \\
Unimodal (120 min) & 28 & 10 & 9 & 9 \\
$100 \%$ Synchrony (120 min) & 30 & $* 23$ & 2 & 5 \\
$100 \%$ Synchrony (60 min) & 30 & 1 & 5 & 11 \\
BSTE (120 min) & 30 & 15 & 10 & 5 \\
\hline
\end{tabular}

$\mathrm{p}<.05$ (chi-square test) 
Table $3 b$

Preference Scores for the Control Group in Experiment 1

\begin{tabular}{llccc} 
& & & \multicolumn{2}{c}{ Preference Category } \\
\cline { 3 - 5 } Prenatal Condition & $\mathrm{N}$ & Area 1 & Area 2 & No Preference \\
\hline $\begin{array}{l}\text { Controls } \\
\text { (No prenatal stimulation) }\end{array}$ & 31 & 5 & 16 & 10 \\
\hline
\end{tabular}

These results converge with previous findings on prenatal perceptual learning in bobwhite quail (Honeycutt \& Lickliter, 2001; Lickliter, Bahrick, and Honeycutt, 2002; Lickliter \& Hellewell, 1992; Sleigh, Columbus, \& Lickliter, 1998). They also demonstrate that bobwhite quail neonates previously trained with prenatal exposure to a bimodally synchronous maternal call can transfer their perceptual learning skills to postnatal life and discriminate a familiar unimodal call from a novel unimodal maternal call at testing. This is evident because bobwhite quail embryos required $240 \mathrm{~min}$ of unimodal prenatal exposure to a maternal call in order to learn the call. However, when prenatal exposure to the maternal call was bimodal and temporally synchronous, 120 min or $60 \mathrm{~min}$ of prenatal exposure was sufficient to foster perceptual learning of the familiarized maternal call. Thus, results of Experiment 1 support the hypothesis that temporal synchrony is a key component for the facilitation of prenatal perceptual learning with respect to unimodal auditory stimulation.

In addition, these results indicate that amodal equivalence in the absence of temporal synchrony is not sufficient to facilitate prenatal learning with respect to unimodal auditory stimulation. In this case, a transfer of prenatal training was not evident because embryos receiving 120 min of exposure to Bimodal Sequential Temporal 
Equivalence (BSTE) did not demonstrate did not discriminate the familiar unimodal auditory call from the novel unimodal auditory call at testing, again suggesting that temporal synchrony plays a primary role in facilitating perceptual learning, with respect to unimodal auditory stimulation, during late prenatal development.

One possible explanation for the facilitative effects of the $100 \%$ Synchrony group was that it provided more overall stimulation than the Unimodal or BSTE groups. In other words, temporally synchronous stimulation provides two concurrent sources of stimulation, one from the auditory modality and one from the visual modality, whereas unimodal or BSTE provides only one modality of stimulation at a time. However, there is evidence suggesting that overall amount of stimulation is likely not a factor contributing to facilitated prenatal learning. For example, Lickliter, Bahrick, and Honeycutt (2002) conducted an experiment in which embryos were exposed to an audio-visual asynchronous condition. In this experiment, two concurrent sources of stimulation, auditory and visual, were available but did not facilitate prenatal perceptual learning, with respect to unimodal stimulation (see also Bahrick \& Lickliter, 2000 for an example with human infants). Thus, it appears that overall amount of stimulation is not a likely explanation for the facilitative effects of intersensory redundancy; rather it appears that the particular temporal configuration of the multiple sources of stimulation contributes to its effectiveness, especially when the auditory and visual modalities are temporally synchronized. However, it is not known how much temporal synchrony is sufficient for the facilitation of prenatal perceptual learning. Specifically, can a single temporally synchronous note be sufficient for the facilitation of prenatal perceptual learning with respect to unimodal stimulation? The following experiment was designed to explore this issue. 
Experiment 2a: Exploring the Sufficient features for the Facilitation of Prenatal Perceptual Learning: Amount of Temporally Synchronous Notes

In Experiment 1, the 100\% Synchrony group contained temporally synchronous notes throughout the stimulus presentation. That is, a temporally synchronous flash occurred on every note of each call burst (e.g., there are 5 notes per call burst). Such a continuous occurrence of temporally synchronous notes is likely to increase the frequency of enhanced neural responses (reviewed in Stein \& Meredith, 1993) which would, in turn, enhance attention and learning. The goal of this experiment was to determine whether a single temporally synchronous note would work just as well to facilitate prenatal perceptual learning with respect to unimodal stimulation. Unlike experiment 1, in which the $100 \%$ Synchrony group contained a temporally synchronous note on every note of the five note call burst, for this experiment, only one temporally synchronous note occurred for every 5 notes in a call burst. Thus, this experiment exposed embryos to stimulation that contained temporally synchronous notes that occurred only $20 \%$ of the time.

In addition, the temporal distribution of synchronous notes was varied for each prenatal condition. The notion behind having various temporal distributions of synchronous notes was based on previous research demonstrating that young infants are sensitive to temporal patterns of visual stimulation (Lewkowicz, 1985). Thus, to control for the possibility that periodicity, instead of synchrony, could be aiding in the facilitation of prenatal learning, the temporal distribution of synchronous notes was varied across prenatal conditions. This allowed for assessments based on the amount of temporally synchronous notes made available (and controlled for the contribution of pattern detection). Given the apparent salience of temporal synchrony during early development 
(see Lewkowicz, 2000), it was hypothesized that stimulation that contained temporal synchrony just $20 \%$ of the time would be sufficient to facilitate prenatal perceptual learning, with respect to unimodal stimulation, regardless of temporal distribution.

\section{Method}

For this experiment, each group was exposed to the same individual maternal call variants (call A and call B) used in the previous experiment. However, groups received a synchronous flash of light only $20 \%$ of the time and in varying temporal distributions. This experiment was composed of six experimental groups: (1) an Asynchronous Flash group $(\mathrm{N}=30)$, received a flash of light 0.9 seconds prior to the onset of a maternal call burst (Figure 5a) and served as a control for the possibility that a flash of light just prior to the call burst could produce an exogenous shift of attention to the maternal call, (2) a $20 \%$ Variable Synchrony group $(\mathrm{N}=29)$, received exposure to a maternal call paired with a single burst of light randomly synchronized to any of the five notes of the call burst (Figure 5b). In other words, embryos in this condition received auditory exposure to the entire call burst with a single temporally synchronous audio-visual note to one of the five notes of the maternal call, (3) a 20\% Onset Synchrony group ( $=31$ received a single burst of light synchronized to the onset (first note) of the call burst (Figure 5c), (4) a 20\% Middle Synchrony group $(\mathrm{N}=30)$ received a single burst of light synchronized at the middle ( $3^{\text {rd }}$ note) of the call burst (Figure 5d), (5) a 20\% Offset Synchrony group ( $\mathrm{N}=$ 32 received a single burst of light synchronized to the offset (last note) of each call burst (Figure 5e), and (6) a 20\% Every $5^{\text {th }}$ Burst Synchrony group $(\mathrm{N}=29)$ received prenatal exposure to the same maternal call however every $5^{\text {th }}$ call burst was temporally synchronized with the light (Figure 5f). The inter-burst interval for all prenatal 
stimulation conditions was $3.4 \mathrm{sec}$ in duration. To assess whether prenatal perceptual learning occurred, embryos from all groups received 120 min of total stimulation time and were then tested at $24 \mathrm{hr}$ following hatching in a 5 min simultaneous-choice test between the familiarized maternal call and a novel variant of the bobwhite maternal call.

Figure 5

Schematic Representation of the Various Audio-Visual Configurations of the Maternal Call used in Experiment 2a. The letters A and V represent the auditory and visual modalities, respectively. The numbers represent each note in the 5-note call burst. The duration of each call burst is 3 seconds and the duration of the inter-burst-interval is $2.4 \mathrm{~s}$.

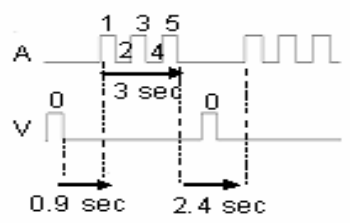
(a) Asynctronous Flash
(120 min)

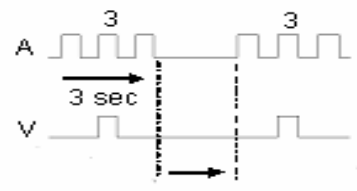

$2.4 \mathrm{sec}$

(d) $20 \%$ Middle Synchrory

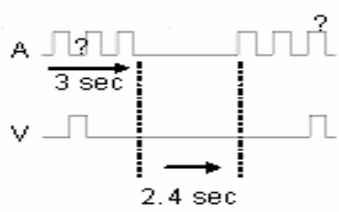

(b) $20 \%$ Variable Synchrony

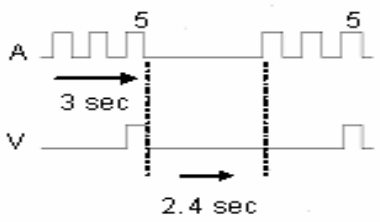

(e) $20 \% 0$ ffset (120 min)

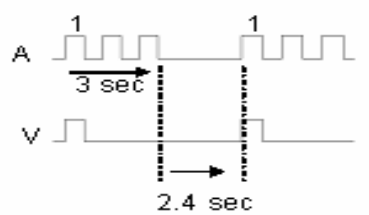

(c) $20 \%$ Onset Synchrony (120 min)

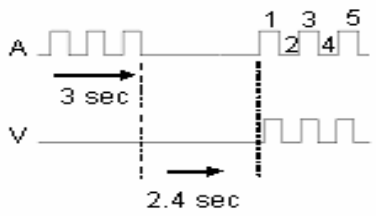

(f) $20 \%$ Every $5 \mathrm{~m}$ Synchrony

\section{Results and discussion}

Results for this experiment are illustrated in Figure 6. One-sample t-tests were performed on the proportion of total duration time (PTDT) spent in the approach area with the familiar call against the chance value of .50. Results revealed that embryos from the 20\% Variable Synchrony (120 min) group showed a greater PTDT to the familiar call at testing $t(28)=4.624, \mathrm{p}=.000$. Likewise, embryos from the $20 \%$ Onset Synchrony $(120$ 
min) and 20\% Middle Synchrony (120 min) groups showed a greater PTDT spent in the approach area with the familiar call at testing, $t(30)=2.082, \mathrm{p}=.046$ and $t(29)=3.396$, $\mathrm{p}=.002$, respectively (Table 4 ). A one-way analysis of variance compared the mean PTDT to the familiar call of all groups. This test was found to be statistically nonsignificant at an alpha level of $.05, \mathrm{~F}(2,87)=1.179, \mathrm{p}=.312$, indicating that the mean PTDT for each of these groups did not differ significantly between groups.

Figure 6

Mean Proportion of Total Duration Time for the Familiar Call in Experiment 2a

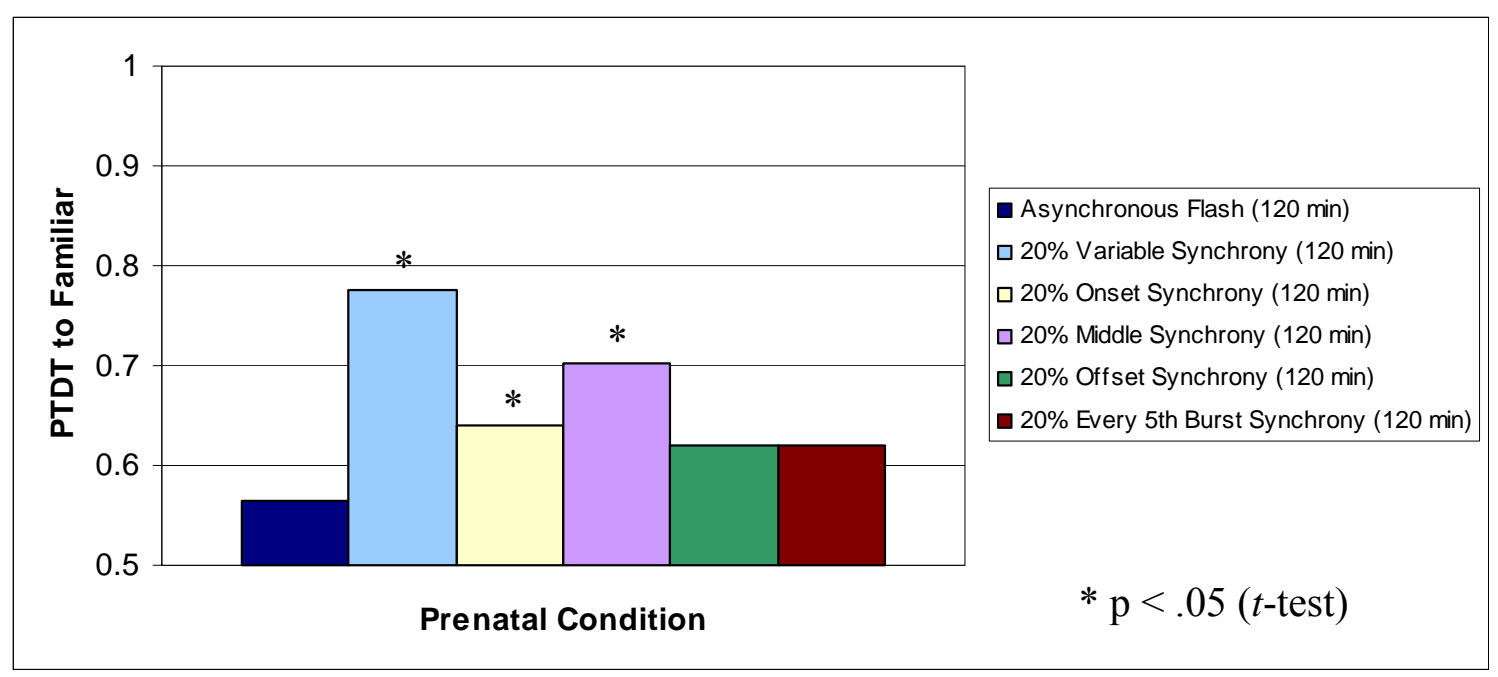


Table 4

Mean Proportions of Total Duration Time and Standard Deviations (in seconds) for the Familiar Call for Experiment 2a

\begin{tabular}{lcc} 
Asynchronous Flash $(120 \mathrm{~min})$ & $\mathrm{x}=.564$ & $(\mathrm{SD}=.364)$ \\
$20 \%$ Variable Synchrony $(120 \mathrm{~min})$ & $\mathrm{x}=.776$ & $(\mathrm{SD}=.321)^{*}$ \\
$20 \%$ Onset Synchrony $(120 \mathrm{~min})$ & $\mathrm{x}=.640$ & $(\mathrm{SD}=.374)^{*}$ \\
$20 \%$ Middle Synchrony $(120 \mathrm{~min})$ & $\mathrm{x}=.703$ & $(\mathrm{SD}=.328)^{*}$ \\
$20 \%$ Offset Synchrony $(120 \mathrm{~min})$ & $\mathrm{x}=.619$ & $(\mathrm{SD}=.360)$ \\
$20 \%$ Every $5^{\text {th }}$ Burst Synchrony $(120 \mathrm{~min})$ & $\mathrm{x}=.588$ & $(\mathrm{SD}=.340)$ \\
\hline$* \mathrm{p}<.05(t$-test $)$ & &
\end{tabular}

Table 5

Means and Standard Deviations of Duration Scores (in seconds) for Experiment 2a

\begin{tabular}{|c|c|c|c|}
\hline \multirow[b]{2}{*}{ Prenatal Condition } & \multirow[b]{2}{*}{$\mathrm{N}$} & \multicolumn{2}{|c|}{ Duration } \\
\hline & & Familiar Call & Novel Call \\
\hline Asynchronous Flash (120 min) & 30 & $\begin{array}{c}101.81 \\
(94.22)\end{array}$ & $\begin{array}{c}52.36 \\
(55.44)\end{array}$ \\
\hline $20 \%$ Variable Synchrony (120 min) & 29 & $\begin{array}{l}123.74^{*} \\
(75.14)\end{array}$ & $\begin{array}{c}30.59 \\
(55.76)\end{array}$ \\
\hline 20\% Onset Synchrony (120 min) & 31 & $\begin{array}{c}65.23 \\
(47.86)\end{array}$ & $\begin{array}{c}44.88 \\
(59.42)\end{array}$ \\
\hline 20\% Middle Synchrony (120 min) & 30 & $\begin{array}{l}85.43 * \\
(60.66)\end{array}$ & $\begin{array}{c}33.63 \\
(39.34)\end{array}$ \\
\hline $20 \%$ Offset Synchrony (120 min) & 32 & $\begin{array}{c}97.51 \\
(87.08)\end{array}$ & $\begin{array}{c}52.99 \\
(75.90)\end{array}$ \\
\hline $20 \%$ Every $5^{\text {th }}$ Burst Synchrony (120 min) & 29 & $\begin{array}{c}64.89 \\
(47.92)\end{array}$ & $\begin{array}{c}41.50 \\
(47.06)\end{array}$ \\
\hline
\end{tabular}

$* \mathrm{p}<.05$ (Wilcoxon signed-ranks test) 
Table 6

Preference Scores for Conditions in Experiment 2a

\begin{tabular}{lcccc}
\hline & & \multicolumn{3}{c}{ Preference Category } \\
\cline { 3 - 5 } Prenatal Condition & $\mathrm{N}$ & Familiar & Non Familiar & No Preference \\
\hline Asynchronous Flash $(120 \mathrm{~min})$ & 30 & 12 & 8 & 10 \\
$20 \%$ Variable Synchrony (120 min) & 29 & $* 23$ & 4 & 2 \\
$20 \%$ Onset Synchrony (120 min) & 31 & $* 18$ & 8 & 5 \\
$20 \%$ Middle Synchrony (120 min) & 30 & $* 19$ & 7 & 4 \\
$20 \%$ Offset Synchrony (120 min) & 32 & $* 18$ & 7 & 6 \\
$20 \%$ Every $5^{\text {th }}$ Synchrony (120 min) & 29 & 13 & 10 & 6 \\
\hline$* \mathrm{p}<.05$ (chi-square test) & & & & 7
\end{tabular}

A Wilcoxon signed-rank test was applied to the duration measures and revealed that embryos exposed to the 20\% Variable (120 min) group and the 20\% Middle Synchrony (120 min) group had significantly longer durations to the approach area containing the familiar call, $\mathrm{z}=-3.362, \mathrm{p}=.001$ and $\mathrm{z}=-2.54, \mathrm{p}=.011$. Contrary to the primary analysis, the results of the $20 \%$ Onset Synchrony (120 min) group did not show a statistically significant difference in duration to the familiar call, $\mathrm{z}=-1.627, \mathrm{p}=.104$. In addition, a Chi-square goodness-of-fit test was applied to the preference scores for the familiar call for each group and found to be statistically significant at an alpha level of .05 for the $20 \%$ Variable synchrony (120 min), 20\% Onset Synchrony (120 min), 20\% Middle Synchrony (120 min), and 20\% Offset Synchrony (120 min) groups, $\chi^{2}(2, \mathrm{~N}=$ $29)=27.793 ; p=.000, \chi^{2}(2, N=31)=8.968 ; p=.011, \chi^{2}(2, N=30)=12.600 ; p=.002$ 
and $\chi^{2}(2, \mathrm{~N}=32)=7.563 ; \mathrm{p}=.023$, respectively. The observed preference scores can be found in Table 6.

In this experiment, the results of the One Sample $t$ test, the Chi-square test, and Wilcoxon test did not correspond. In particular, the Chi square test revealed a significant number of individual preferences for the familiar call in the 20\% Offset Synchrony (120 min) group however the One-sample $t$ test and Wilcoxon test did not find the 20\% Offset Synchrony (120 min) group significant. Based on the established criterion (see Data Analyses section) the overall interpretation is that of non-facilitated prenatal perceptual learning, with respect to unimodal auditory stimulation, for the $20 \%$ Offset Synchrony (120 min) group.

Nonetheless, an interesting finding of this experiment is the relative effectiveness of just a single temporally synchronous note within a call burst. It appears that prenatal training with bimodal (audio-visual) stimulation containing just a single temporally synchronous note is sufficient to transfer to the postnatal discrimination of the same unimodal (auditory) familiarized call from a unimodal (auditory) novel call. Specifically, the 20\% Variable Synchrony (120 min), 20\% Onset Synchrony (120 min), and 20\% Middle Synchrony (120 min) groups showed facilitated prenatal perceptual learning, with respect to unimodal auditory stimulation. In contrast, the 20\% Every 5th Synchrony (120 min) group did not show facilitated prenatal perceptual learning, with respect to unimodal auditory stimulation, even though the proportion of temporally synchronous notes made available was the same as the other groups. This suggests that the temporal distribution of the temporally synchronous notes may play a role in facilitated prenatal perceptual learning. One aspect of this episodic temporal synchrony that needs further exploration is 
its timing. That is, when in the stimulus event is temporal synchrony most effective at facilitating prenatal learning with respect to unimodal stimulation? Given that the $20 \%$ Variable Synchrony (120 min), 20\% Onset Synchrony (120 min), and 20\% Middle Synchrony (120 min) groups showed significant durations to the familiar call, little can be said from the results of the current experiment as to when the occurrence of a synchronous note is most effective. The following experiment assessed this question.

Experiment 2b: Exploring the Sufficient Features for the Facilitation of Prenatal Perceptual Learning: Timing of Temporally Synchronous Notes

This experiment explored the role that timing of a single temporally synchronous note plays in the facilitation of prenatal perceptual learning. In a previous investigation of prenatal learning with quail embryos, Lickliter, Bahrick, and Markham (2006) demonstrated that embryos' attention can be educated to the amodal stimulus properties of a unimodal maternal call when a relatively brief exposure to the same bimodally redundant call precedes it (e.g., $6 \mathrm{hr}$ of a temporally synchronous call followed by $18 \mathrm{hr}$ of a unimodal call). However, when the stimulation was presented to embryos in the reverse order (e.g., $18 \mathrm{hr}$ of a unimodal call followed by $6 \mathrm{hr}$ of a temporally synchronous call) facilitated prenatal learning did not occur. Thus, it appears that timing of presentation of temporally synchronous audio-visual stimulation can modulate attention to subsequent unimodal auditory stimulation such that if temporal synchrony precedes the unimodal event then fostering of attention to the amodal stimulus properties occurs. However, if temporal synchrony follows the unimodal event, then attention to the unimodal event is attenuated. Based on these earlier findings, a similar effect was predicted in this experiment. It was hypothesized that a single temporally synchronous 
note would be most effective at facilitating prenatal perceptual learning when it occurred at the onset of the call burst by fostering attention to the amodal properties in the entire stimulus burst.

\section{Method}

Only those prenatal stimulation conditions that showed facilitated prenatal perceptual learning, with respect to unimodal stimulation, in Experiment 2 a were used as prenatal conditions in this experiment. However, embryos from all groups in this experiment received exposure for just $10 \mathrm{~min} / \mathrm{hr}$ for $6 \mathrm{hr}$ (60 min total stimulation time) prior to hatching. Thus, this experiment was composed of three experimental groups: (1) a 20\% Variable Synchrony (60 min) group ( $\mathrm{N}=28)$, (2) a 20\% Onset Synchrony (60 $\min )$ group $(\mathrm{N}=28)$, and (3) a 20\% Middle Synchrony (60 min) group $(\mathrm{N}=29)$. All chicks were tested at $24 \mathrm{hr}$ following hatching in a 5 min simultaneous-choice test between the familiarized maternal call and a novel variant of the bobwhite maternal call.

\section{Results and discussion}

Results for this experiment are illustrated in Figure 7. One-sample t-tests were performed on the proportion of total duration time (PTDT) spent in the approach area with the familiar call against the chance value of .50. Results revealed that only embryos from the 20\% Onset Synchrony (60 min) group showed a greater PTDT to the familiar call at testing $\mathrm{t}(27)=3.516, \mathrm{p}=.002$ (Table 7$)$. A one-way analysis of variance compared the mean PTDT to the familiar call of all groups. This test was found to be statistically non-significant at an alpha level of .05, F $(2,77)=2.147, \mathrm{p}=.124$, indicating that the mean PTDT for each of these groups did not differ. 
Figure 7

Mean Proportion of Total Duration Time for the Familiar Call in Experiment $2 b$

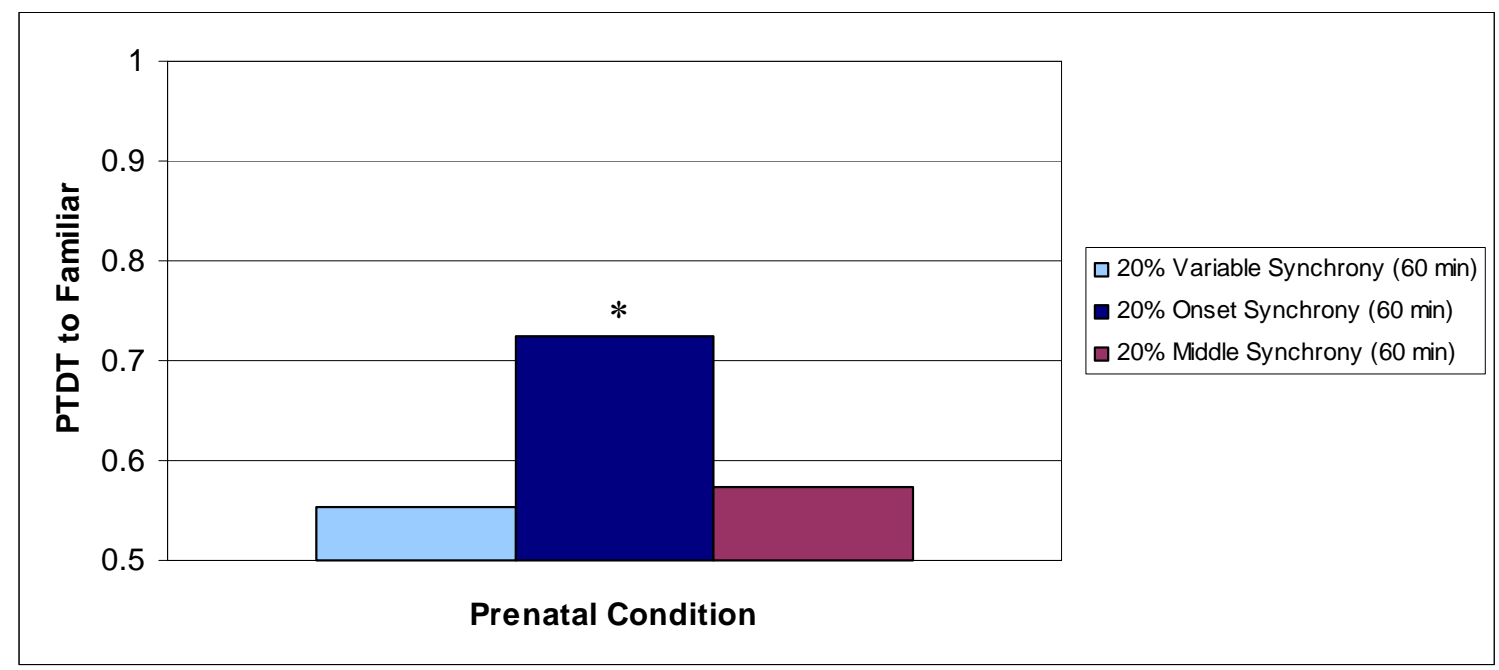

Table 7

Mean Proportions of Total Duration Time and Standard Deviations (in seconds) for the Familiar Call in Experiment 2b

$\begin{array}{lll}20 \% \text { Variable Synchrony }(60 \mathrm{~min}) & \mathrm{x}=.554 & (\mathrm{SD}=.320) \\ 20 \% \text { Onset Synchrony }(60 \mathrm{~min}) & \mathrm{x}=.725 & (\mathrm{SD}=.339)^{*} \\ 20 \% \text { Middle Synchrony }(60 \mathrm{~min}) & \mathrm{x}=.574 & (\mathrm{SD}=.450)\end{array}$

$* \mathrm{p}<.05(t$-test $)$

A Wilcoxon signed-rank test was applied to the duration measures and revealed that embryos from the $20 \%$ Onset Synchrony (60 min) group also had significantly longer durations to the approach area containing the familiar call, $\mathrm{z}=-2.437, \mathrm{p}=.015$ (Table 8). In addition, a Chi-square goodness-of-fit test revealed that only the $20 \%$ Onset Synchrony (60 min) group showed a statistically significant preference for the familiar 
call at testing, $\chi^{2}(2, N=28)=18.500 ; p=.000$. The observed preference scores can be found in Table 9.

Table 8

Means and Standard Deviations of Duration Scores (in seconds) for Experiment 2b

\begin{tabular}{lccc}
\hline Prenatal Condition & N & Familiar Call & Novel Call \\
\hline $20 \%$ Variable Synchrony (60 min) & 28 & 68.27 & 60.44 \\
& & $(49.36)$ & $(62.61)$ \\
$20 \%$ Onset Synchrony (60 min) & 28 & $88.83 *$ & 38.06 \\
& & $(63.88)$ & $(58.99)$ \\
$20 \%$ Middle Synchrony (60 min) & 29 & 90.40 & 56.73 \\
& & $(97.51)$ & $(62.93)$ \\
\hline
\end{tabular}

$* \mathrm{p}<.05$ (Wilcoxon signed-ranks test)

Table 9

Preference Scores for Conditions in Experiment 2b

\begin{tabular}{lcccc}
\hline & & \multicolumn{3}{c}{ Preference Category } \\
\cline { 3 - 5 } Prenatal Condition & $\mathrm{N}$ & Familiar & Non Familiar & No Preference \\
\hline $20 \%$ Variable Synchrony (60 min) & 28 & 13 & 9 & 6 \\
$20 \%$ Onset Synchrony (60 min) & 28 & $* 20$ & 5 & 3 \\
$20 \%$ Middle Synchrony (60 min) & 29 & 13 & 14 & 2 \\
\hline
\end{tabular}

$* \mathrm{p}<.05$ (chi-square test)

Taken together, these results demonstrate that the most effective position for a temporally synchronous note to occur for the transfer of prenatal training to postnatal perceptual discrimination of a familiar versus a novel unimodal call is at the onset of the call burst. This is indicative by the fact that only the $20 \%$ Onset Synchrony (60 min) 
group showed significantly greater PTDTs, durations, and number of preferences to the approach area with the familiar call at 24 testing. In fact, the 20\% Onset Synchrony (60 $\mathrm{min}$ ) group was just as effective as the $100 \%$ Synchrony $(60 \mathrm{~min})$ group from Experiment 1 at facilitating prenatal learning. Thus, prenatal exposure to onset synchrony can facilitate perceptual learning four times faster than prenatal exposure to unimodal stimulation. This finding highlights the powerful role that timing plays on the effectiveness of bimodally redundant stimulation. Quail embryos appear to be highly sensitive to the timing of presentation of stimulation (see also Honeycutt \& Lickliter, 2001) and appear to benefit from its occurrence at the onset of the multisensory event.

\section{General Discussion}

In this study, the transfer of perceptual learning and discrimination from the prenatal to the postnatal period was facilitated by training bobwhite quail embryos with synchronous audio-visual stimulation occurring just at the onset of each call burst. As a result, 24-hr-old neonates were able to effectively discriminate a familiar call from a novel call both presented in the auditory modality with reduced prenatal exposure amounts relative to what is typically needed with unimodal prenatal exposure. These findings are consistent with previous studies demonstrating that prenatal perceptual training in bobwhite quail can be enhanced when embryos are exposed to temporally synchronous redundant audio-visual stimulation (Lickliter, Bahrick, \& Honeycutt, 2002, 2004). However, what distinguishes this study from previous work is that the current findings advance our understanding of the features of temporally synchronous stimulation that are most effective in facilitating selective attention and perceptual learning, with respect to unimodal stimulation, during prenatal development. To my knowledge, no 
studies have specifically assessed the role of amount and timing of the amodal property of temporal synchrony in the deployment of attention and perceptual learning during prenatal development.

Experiment 1 explored if previous findings of facilitated prenatal training, with respect to unimodal auditory stimulation, was based on the detection of temporal synchrony or if intermodal equivalence could also aid in facilitating prenatal perceptual learning. To assess this, Bimodal Sequential Temporal Equivalence (BSTE) was created. BSTE provided embryos with sequential auditory and visual stimulation in which the same amodal properties (rate, duration, and rhythm) were made available across modalities. It was predicted that if embryos are sensitive to intersensory equivalence, then those chicks prenatally exposed to BSTE should show a preference for the familiar call at testing. However, if embryos are not sensitive to intersensory equivalence then no such indication of perceptual learning would result.

Results revealed that embryos that received exposure to a unimodal maternal call (e.g., the Unimodal Group) required 240 total min of exposure time to learn the call. Embryos that received exposure to the 100\% Synchrony group demonstrated facilitated perceptual learning with respect to unimodal stimulation, in that they only required 60 min of total exposure time to the maternal call. In contrast, embryos that received 120 min of BTSE did not learn the call, suggesting that intersensory sequential equivalence was not sufficient for facilitated prenatal perceptual learning, with respect to unimodal stimulation. These results also suggest that temporal synchrony is a powerful feature in the deployment of attention and perceptual learning and support the hypothesis that 
temporal synchrony is a critical feature for facilitated prenatal perceptual learning with respect to unimodal stimulation.

Experiment 1 also provides support for the argument raised by Lewkowicz and Kraebel (2004), that early developing organisms may not be able to perceive the temporal pattern of amodal properties when only available in a single sensory modality. Thus far, these results provide continued support for the notion that intersensory redundancy is the feature that makes amodal properties salient to organisms during early development (Bahrick \& Lickliter, 2000). As indicated from the results of the BSTE group, it appears that embryos are not sensitive to sequential intersensory equivalence. This was a difficult task considering that embryos would have had to retain the temporal amodal properties of the auditory stimulus in memory and detect those same amodal features in the subsequently presented visual modality. Interestingly, human infant studies on sensitivity to intersensory equivalence report these abilities emerging between 4 and 8 months of age (e.g., Allen, Walker, Symonds, \& Marcell, 1977; Mendelson \& Ferland, 1982; Lewkowicz, 1992). Thus, it is likely that the ability to detect intersensory sequential equivalence is dependent upon perceptual experience.

Experiment 2a explored whether limited amounts of temporal synchrony was sufficient for facilitated prenatal perceptual learning. In order to investigate this question, stimulation was created that contained temporally synchronous notes occurring only $20 \%$ of the time. Recall that the $100 \%$ Synchrony condition in Experiment 1 contained a temporally synchronous note on all five notes of each call burst. However, for Experiment 2a, stimulation was provided to embryos that contained only 1 temporally synchronous note per call burst. Hence, each prenatal condition in Experiment 2a 
provided synchrony $20 \%$ of the total exposure time. The main question of interest was whether this limited temporal synchrony would be sufficient to facilitate prenatal perceptual learning with respect to unimodal stimulation. It was important to control for the possibility that embryos' prenatal perceptual learning could result from sensitivity to patterns of periodicity (Lewkowicz, 1985). Therefore, each prenatal condition contained a different temporal distribution of the temporally synchronous notes. In addition, an asynchronous condition, in which a flash or light occurred just 0.9 seconds prior to every call burst, was also created to control for whether it was the light that was producing an exogenous shift of the embryos' attention to the remainder of the call burst.

As expected, the Asynchronous Flash group did not demonstrate facilitated prenatal perceptual learning because there were no temporally synchronous notes available in the stimulus event. The result of the Asynchronous Flash group provides additional support for the notion that temporal synchrony is a critical feature for the facilitation of prenatal perceptual learning. This is not to suggest the there is no exogenous shift of attention occurring. In fact, the literature suggests that the same neurophysiologic mechanisms mediating overt orientation behaviors also mediate covert (exogenous) shifts of attention (Desimone, Wessinger, Thomas, \& Schneider, 1992; Robinson \& Kertzman, 1995; Stein, Wallace, \& Meredith, 1995; Thompson \& Masterton, 1978). Thus, it is likely that temporal synchrony is also a critical component for exogenous attention during prenatal development.

An important additional finding of this experiment was that even small amounts of temporally synchronous notes were sufficient to facilitate prenatal perceptual learning, with respect to unimodal stimulation, as evidenced by the fact that embryos exposed to 
the $20 \%$ Variable Synchrony (120 min) condition, the 20\% Onset Synchrony (120 min) condition, and the $20 \%$ Middle Synchrony (120 min) condition all demonstrated facilitated prenatal perceptual learning. However, the 20\% Offset Synchrony (120 min) and the $20 \%$ Every $5^{\text {th }}$ Synchrony $(120 \mathrm{~min}$ ) groups did not show facilitated prenatal perceptual learning. Although these groups also contained temporally synchronous notes $20 \%$ of total exposure time, it appears that certain temporal distributions of synchronous notes were not effective and raised the possibility that the timing of occurrence of synchrony could be a contributing factor to facilitated prenatal perceptual learning.

Experiment $2 \mathrm{~b}$ evaluated this question regarding the relationship between timing of occurrence of a temporally synchronous note and the facilitation of prenatal perceptual learning with respect to unimodal stimulation. Several steps were taken to explore this issue. First, embryos were exposed to only those prenatal conditions that showed enhanced prenatal perceptual learning, with respect to unimodal stimulation, in Experiment 2a. Thus, only the 20\% Variable Synchrony, 20\% Onset Synchrony, and $20 \%$ Middle Synchrony groups were evaluated. Second, the total prenatal exposure time was reduced to only $60 \mathrm{~min}$ (half the exposure time of Experiment 2a). It was predicted that stimulation that contained a temporally synchronous note at the onset of the call burst would be the most effective at facilitating prenatal perceptual learning with respect to unimodal stimulation. As predicted, results from Experiment $2 \mathrm{~b}$ revealed that only chicks that received 20\% Onset Synchrony (60 $\mathrm{min}$ ) prenatally showed a significant preference for the familiarized call at testing. These results provide support for the notion that synchrony occurring at the onset of a stimulus is particularly salient in grabbing embryos' attention to the amodal features of sensory stimulation. 
Thus, critical features in the facilitation of prenatal perceptual learning with respect to unimodal stimulation were identified in this study: (1) for the facilitation of prenatal perceptual learning to occur with respect to unimodal stimulation, the temporally synchronous overlap of auditory and visual modalities is a critical feature, (2) for the facilitation of prenatal perceptual learning to occur with respect to unimodal stimulation, a single temporally synchronous note is sufficient, and (3) a single temporally synchronous note is most effective when it occurs at the onset of a stimulus event. In fact, the facilitative effects of a single temporally synchronous note at the beginning of the call burst were comparable to those supported by $100 \%$ synchronous stimulation. Taken together, these findings indicate that precocial avian embryos are remarkably sensitive to the presence, amount, and timing of temporal synchrony during the late stages of prenatal development.

Perhaps the most parsimonious explanation for these facilitative effects is that neural enhancement may be occurring in multisensory regions of the avian brain, fostering enhanced orientation and selective attention to the call features. Neural enhancement has been demonstrated to occur in a multisensory region of the mammalian midbrain known as the superior colliculus (SC). These SC neurons show significantly enhanced discharges to temporally synchronous bisensory stimulation which exceed those of the most effective responses elicited by modality-specific stimuli (Stein \& Meredith, 1993). Previous studies on multisensory enhancement have demonstrated this effect in cats, an altricial species (Wallace \& Stein, 1997). However, with its sensory systems poorly developed at birth, altritial cats need a great deal of postnatal maturation before neural enhancement effects are observed (Norton, 1974; Stein, Labos, \& Kruger, 
1973a, 1973b; Stein \& Gallagher, 1981; Wallace \& Stein, 1997). Recently, however, multisensory enhancement has been demonstrated in the $\mathrm{SC}$ in the newborn monkey (Wallace \& Stein, 2001). Contrary to the undeveloped sensory systems of the newborn cat, the monkey's sensory systems are functional at birth (Boothe, Dobson, \& Teller, 1985; Carlson, 1984; King, Fobes, \& Fobes 1974). Thus, one can argue that multisensory enhancement effects can differ across species, depending on a species' rate of maturation (i.e., altritial or precocial). Although neural enhancement has yet to be reported in the brain of the precocial avian embryo, the rapid development of the avian auditory and visual systems makes this a likely possibility (Zeigler \& Bischof, 1993).

This leads us to the question, why would onset synchrony be the most effective form of episodic temporal synchrony? One could postulate that the effectiveness of the $20 \%$ Onset Synchrony condition was a multiplicative effect resulting from the first note containing one of the weakest auditory signals in the call burst. As stated in Stein and Meredith (1993), "maximal enhancement occurs with minimally effective stimuli" (p. 117). Indeed, Stein and Meredith (1993) provide evidence that an inverse relationship exists between the effectiveness of unimodal stimuli and responses evoked. That is, the synchronous overlap of two weak unimodal inputs produces the greatest enhancements. Upon examination of the spectrogram of both bobwhite maternal calls (Figure 2), one can see that the first note of the call burst produces one of the lowest frequencies throughout the $3 \mathrm{sec}$ call burst. Thus, a flash of light synchronized at this particular moment, when the auditory signal is weakest, could actually be the point of greatest multisensory enhancement. This would also explain why the synchronous flash of light occurring at other notes in the call (where the unimodal signal is a little stronger) did not facilitate 
prenatal perceptual learning, with respect to unimodal stimulation, with the same effectiveness (i.e., with 60 min of prenatal exposure time).

It is important to note that quail embryos in this study received exposure to stimuli they would normally not receive in natural settings (patterned pulsing light). Although this allowed for a high degree of experimental control during prenatal development, future experiments should make efforts to use more ecologically valid stimuli. One feasible approach would be to provide embryos with temporal synchrony in other modalities such as vestibular (vibration) and auditory (call). Exposing embryos to temporal synchrony in these two modalities could shed light on how temporal properties influence intersensory development under more ecologically appropriate prenatal conditions.

Nonetheless, these results suggest intriguing implications for the identification of potential precursors to attention disorders in young children. For example, Attention Deficit Hyperactivity Disorder (ADHD) is characterized by the relationship between increased levels of inattention, hyperactivity, and impulsivity (American Psychiatric Association [APA], 2000). Thus, one area of exploration would be to assess the relationship between the timing of occurrence of temporal synchrony and an embryo's state of arousal. Does the timing of occurrence of temporal synchrony modulate physiological arousal? This may help us in understanding what sensory features of the prenatal environment may contribute to elevated states of arousal in children with ADHD. Previous comparative work has provided initial evidence that the temporal configuration of multisensory stimulation can modulate bobwhite quail embryos' level of arousal (Reynolds \& Lickiter, 2002). The present investigation highlights the importance 
of continued research in this domain. A more fine grained analysis of the effects of episodic temporal synchrony could shed light on the interplay between attention and arousal that is critical for normal functioning. Thus, the potential for this area of comparative research to influence the type of questions asked in human research is substantial, particularly in addressing the prenatal precursors of attentional disturbances.

This is not to suggest that bobwhite quail embryos can serve as an animal model of prenatal attention and perception for humans, or for that matter, of ADHD. However, given the plasticity of the early developing nervous system, this type of comparative work can be useful in the identification of necessary and/or sufficient epigenetic factors contributing to the development of both typical and atypical perception, arousal and attention during early ontogenesis (Gottlieb, 1991; Karmiloff-Smith, 2007; Lickliter, 2000). One step in this direction would be to assess whether modified amounts of temporal synchrony can derail the normal trajectory of developing attentional and arousal systems in fetuses or neonates.

In a more general sense, this study adds to the growing body of work demonstrating that experiential factors during prenatal development play a key role in the organization of perception and learning. The present results sheds some light on bobwhite quail embryos' remarkable sensitivity to temporal synchrony and suggests that this amodal property may be the basis for selective attention, perceptual learning and organization during prenatal development. Consistent with the infant literature (see Lewkowicz 2000 for a review), the present study provides strong evidence that temporal synchrony is one of the most salient stimulus properties for the facilitation of attention and perception during early development. These findings also provide evidence that 
perceptual capacities that might seem innate or hardwired at birth have actually developed through activity dependent processes during prenatal life. For example, temporal synchrony is an amodal property that is ubiquitous across prenatal and postnatal life. Thus, experience with concurrent and synchronous sources of sensory stimulation during prenatal development can prepare the embryo for the transition from an attenuated perceptual world into an array of temporally and spatially coordinated sights and sounds during postnatal development (Lickliter, 2005).

In summary, this study demonstrates that bobwhite quail embryos are highly sensitive to the temporal distribution of the amodal property of temporal synchrony. This sensitivity to the timing of a single temporally synchronous note modulates their ability to attend to stimulation during prenatal development. These findings add to the current body of knowledge regarding the nature of how multisensory stimulation contributes to the emergence, maintenance, and organization of perception during prenatal development (Gottlieb, 1973; Lickliter, 1993). 


\section{REFERENCES}

Alder, S. A., Wilk, A., \& Rovee-Collier, C. (2000). Reinstatement versus reactivation effects on active memory in infants. Journal of Experimental Child Psychology, 75, 93-115.

Allen, T. W., Walker, K., Symonds, L., \& Marcell, M. (1977). Intrasensory and intersensory perception of temporal sequences in infancy. Developmental Psychology, 13, 225-229.

American Psychiatric Association (2000). Diagnostic and statistical manual of mental disorders, 4th edition, text revision. Washington, DC: American Psychiatric Press, Inc.

Bahrick, L. E. (1983). Infants' perception of substance and temporal synchrony in multimodal events. Infant Behavior and Development, 6, 429-451.

Bahrick, L. E. (1987). Infants' intermodal perception of two levels of temporal structure in natural events. Infant Behavior and Development, 10, 387-416.

Bahrick, L. E. (1988). Intermodal learning in infancy: Learning on the basis of two kinds of invariant relations in audible and visible events. Child Development, 59, 197209.

Bahrick, L. E. (1992). Infants' perceptual differentiation of amodal and modality-specific audio-visual relations. Journal of Experimental Child Psychology, 53, 180-199.

Bahrick, L.E. (2001). Increasing specificity in perceptual development: Infants' detection of nested levels of multimodal stimulation. Journal of Experimental Child Psychology, 79, 253-270.

Bahrick, L. E. (2002). Generalization of learning in the three-month-old infants on the basis of amodal relations. Child Development, 73, 667-681.

Bahrick, L. E., Flom, R., \& Lickliter, R. (2002). Intersensory redundancy facilitates discrimination of tempo in 3-month-old infants. Developmental Psychobiology, $41,352-363$.

Bahrick, L. E., \& Lickliter, R. (2000). Intersensory redundancy guides attentional selectivity and perceptual learning in infancy. Developmental Psychology, 36, 190-201. 
Bahrick, L. E., \& Lickliter, R. (2004). Infants' perception of rhythm and tempo in unimodal and multimodal stimulation: A developmental test of the intersensory redundancy hypothesis. Cognitive, Affective and Behavioral Neuroscience, 4, 137-147.

Bahrick, L. E., \& Lickliter, R., \& Flom, R. (2006). Up versus down: The role of intersensory redundancy in the development of infants' sensitivity to the orientation of moving objects. Infancy, 9, 73-96.

Bahrick, L. E., \& Pickens, J. (1994). Amodal relations: The basis for intermodal perception and learning in infancy. In D. J. Lewkowicz \& R. Lickliter (Eds.), The development of intersensory perception: Comparative perspectives (pp. 205-233). Hillsdale, NJ: Erlbaum.

Bahrick, L.E., Walker, A.S., and Neisser, U. (1981). Selective looking by infants. Cognitive Psychology, 13, 377-390.

Boothe, R. G., Dobson, V., \& Teller, D. Y. (1985). Postnatal development of vision in human and nonhuman primates. Annual Review of Neuroscience, 8, 495-545.

Carlson, M. (1984). Development of tactile discrimination capacity in Macaca mulatta. I. Normal infants. Brain Research, 318, 69-82.

Casagrande, V. A., Harting, J. K., Hall, W. C., \& Diamond, I. T. (1972). Superior colliculus of the tree shrew: A structural and functional subdivision into superficial and deep layers. Science, 177, 444-447.

Chen, W. J., Lariviere, N. A., Heyser, C. L., Spear, L. P., \& Spear, N. E. (1991). Agerelated differences in sensory conditioning in rats. Developmental Psychobiology, 24, 307-325.

Cheslock, S. J., Varlinskaya, E. I., High, J. M., \& Spear, N. E. (2003). Higher order conditioning in the newborn rat: Effects of temporal disparity imply infantile encoding of stimulus events. Infancy, 4, 157-176.

Cornell, E. H. (1979). Infants' recognition memory, forgetting, and savings. Journal of Experimental Child Psychology, 28, 359-374.

Desimone, R., Wessinger, M., Thomas, J., \& Schneider, W. (1992). Attentional control of visual perception: Cortical and subcortical mechanisms. Cold Spring Harbor Symposia on Quantitative Biology, 55, 963-971.

Edelman, G. M. (1992). Bright air, brilliant fire. New York: Basic Books. 
Fifer, W. P. \& Moon, C. M. (1995). The effects of fetal experience with sound. In Lecanuet, J., Fifer, W. P., Krasnegor, N. A., \& Smotherman, W. P. (Eds.), Fetal Development: A Psychobiological Perspective, (pp. 351-366). New Jersey: Lawrence Erlbaum Associates.

Fitzpatrick, P., Arsenio, A., \& Torres-Jara, E. R. (2006). Reinforcing robot perception of multi-modal events through repetition and redundancy and repetition and redundancy. Interaction Studies, 7, 171-196.

Fraisse, P. (1982). The adaptation of the child to time. In W. J. Friedman (Ed.), The developmental psychology of time (pp. 113-140). New York: Academic Press.

Gibson, E. J. (1969). Principles of perceptual learning and development. New York: Appleton.

Gibson, E. J., \& Pick, A. D. (2000). An ecological approach to perceptual learning and development. New York: Oxford University Press.

Gottlieb, G. (1973). Introduction to behavioral embryology. In G. Gottlieb (ED.), Behavioral Embryology (pp. 3-45). New York: Academic

Gottlieb, G. (1991). Experiential canalization of behavioral development: Theory. Developmental Psychology, 27, 4-13.

Heaton, M. B., Miller, D. B., \& Goodwin, D. G. (1978). Species-specific auditory discrimination in bobwhite quail neonates. Developmental Psychobiology, 11, 1321 .

Honeycutt, H., \& Lickliter, R. (2001). Order-dependent timing of unimodal and multimodal stimulation affects prenatal auditory learning in bobwhite quail embryos. Developmental Psychobiology, 38, 1-10.

Horder, T. J., \& Mashkas, A. (1982). The developmental programme for retinal embryogenesis with special reference to the chick. Bibliotheca Anatomica, 23, 103-123.

Hultsch, H., Scheuss, F., \& Todt, D. (1999). Auditory-visual stimulus pairing enhances perceptual learning in a songbird. Animal Behaviour, 58, 143-149.

Jay, M. F., \& Sparks, D. L. (1984). Sensorimotor integration in the primate superior colliculus: II. Coordinates of auditory signals. Journal of Neurophysiology, 57, 35-55.

Jiang, W., Jiang, H., \& Stein, B. E. (2002). Two corticotectal areas facilitate multisensory orientation behavior. Journal of Cognitive Neuroscience, 14, 1240-1255. 
Kadunce, D. C., Vaughan, J. W., Wallace, M. T., Benedek, G., \& Stein, B. E. (1997). Mechanisms of within-modality and cross-modality suppression in the visual and auditory representations in the mammalian superior colliculus. Nature, 332, 7376.

Karmiloff-Smith, A. (2007). Atypical epigenesist. Developmental Science, 10, 84-88.

Kahn, A. J. (1973). Ganglion cell formation in the chick neural retina. Brain Research, 63, 285-290.

Kahn, A. J. (1974). An autoradiographic analysis of the time of appearance of neurons in the developing chick neural retina. Developmental Biology, 38, 30-40.

King, J. E., Fobes, J. T., \& Fobes, J. L. (1974). Development of early behaviors in neonatal squirrel monkeys and cotton-top tamarins. Developmental Psychobiology, 7, 97-109.

King, A. J., \& Palmer, A. R. (1985). Integration of auditory visual information in bimodal neurons in the guineapig superior colliculus. Experimental Brain Research, 60, 492-500.

Klein, R. M., Kingstone, A., \& Pontefract, A. (1992). Orienting of visual attention. In K. Rayner (Ed.), Eye movements and visual cognition: Scene perception and reading (pp. 46-65). New York: Springer-Verlag.

Konishi, M. (1973). Development of auditory neuronal responses in avian embryos. Proceedings of the National Academy of Sciences, 70, 1795-1798.

Lawson, K. R. (1980). Spatial and temporal congruity and auditory-visual integration in infants. Developmental Psychology, 16, 185-192.

LaVail, J. H., \& Cowan, W. M. (1971). The development of the chick optic tectum. II. Autoradiographic studies. Brain Research, 28, 421-441.

Lewkowicz, D. J. (1985). Developmental changes in infants' response to temporal frequency. Developmental Psychology, 21, 850-865.

Lewkowicz, D. J. (1986). Developmental changes in infants' bisensory response to synchronous durations. Infant Behavior and Development, 9, 335-353.

Lewkowicz, D. J. (1988). Sensory dominance in infants: II. Ten-month-old infants' response to auditory-visual compounds. Developmental Psychology, 24, 172-182. 
Lewkowicz, D. J. (1992). Infants' responsiveness to the auditory and visual attributes of a sounding/moving stimulus. Perception \& Psychophysics, 52, 519-528.

Lewkowicz, D. J. (1996). Perception of auditory-visual temporal synchrony in human infants. Journal of Experimental Psychology: Human Perception and Performance, 22, 1094-1106.

Lewkowicz, D. J. (2000). The development of intersensory temporal perception: an epigenetic system/limitations view. Psychological Bulletin, 126, 281-308.

Lewkowicz, D. J., \& Kraebel, K. S. (2004). The value of multisensory redundancy in the development of intersensory perception. In G. Calvert, C. Spence, \& B. E. Stein (Eds.), The handbook of multisensory processes (pp. 655-678). Cambridge, MA: MIT Press.

Lickliter, R. (1993). Timing and the development of perinatal perceptual organization. In G. Turkewitz \& D. A. Devenny (Eds.), Developmental time and timing (pp. 105123). Hillsdale, NJ: Erlbaum.

Lickliter, R. (2000). The role of sensory stimulation in perinatal development: Insights from comparative research for care of the high-risk infant. Developmental and Behavioral Pediatrics, 21, 437-447.

Lickliter, R. (2005). Prenatal sensory ecology and experience: Implications for perceptual and behavioral development in precocial birds. In P. Slater, J. Rosenblatt, C. Snowden, T. Roper, H. J. Brockmann, \& M. Naguib (Eds.), Advances in the Study of Behavior, Vol. 35, pp. 235-274. Academic Press, New York.

Lickliter, R., \& Bahrick, L. E. (2000). The development of infant intersensory perception: Advantages of a comparative convergent-operations approach. Psychological Bulletin, 126, 260-280.

Lickliter, R., \& Bahrick, L. E. (2001). The salience of multimodal sensory stimulation in early development: Implications for the issue of ecological validity. Infancy, 2, $447-463$.

Lickliter, R., Bahrick, L. E., \& Honeycutt, H. (2002). Intersensory redundancy facilitates prenatal perceptual learning in bobwhite quail (Colinus virginianus) embryos. Developmental Psychology, 38, 15-23.

Lickliter, R., Bahrick, L.E., \& Honeycutt, H. (2004). Intersensory redundancy enhances memory in bobwhite quail embryos. Infancy, 5, 253-269 
Lickliter, R., Bahrick, L., \& Markham, R.G. (2006). Intersensory redundancy educates selective attention in bobwhite quail embryos. Developmental Science, 9, 604615.

Lickliter, R., \& Hellewell, T. (1992). Contextual determinants of auditory learning in bobwhite quail embryos and hatchlings. Developmental Psychobiology, 25, 1-24.

Lickliter, R., \& Lewkowicz, D.J. (1995). Intersensory experience and early perceptual development: Attenuated prenatal sensory stimulation affects postnatal auditory and visual responsiveness in bobwhite quail chicks. Developmental Psychology, 31, 609-618.

Lyons-Ruth, K. (1977). Bimodal perception in infancy: Response to auditory-visual incongruity. Child Development, 48, 820-827.

Mathers, L. H., \& Ostrach, L. H. (1979). A critical period in the development of tectal neurons in the chick, as revealed by early enucleation. Brain Research, 170, 219230.

McGrath, M., \& Summerfield, Q. (1985). Intermodal timing relations and audio-visual speech recognition by normal-hearing adults. Journal of the Acoustical Society of America, 77, 678-685.

McGraw, C. F., \& McLaughlin, B. J. (1980). Fine structural studies of synaptogenesis in the superficial layers of the chick optic tectum. Journal of Neurocytology, 9, 7993.

Meltzoff, A. N., \& Kuhl, P. K. (1994). Faces and speech: Intermodal processing of biologically relevant signals in infants and adults. In D. J. Lewkowicz \& R. Lickliter (Eds.), The development of intersensory perception: Comparative perspectives (pp. 335-369). Hillsdale, NJ: Erlbaum.

Mendelson, M. J., \& Ferland, M. B. (1982). Auditory-visual transfer in four-month-old infants. Child Development, 53, 1022-1027.

Meredith, M. A., Nemitz, J. W., \& Stein, B. E. (1987). Determinants of multisensory integration in superior colliculus neurons: I. Temporal factors. Journal of Neuroscience, 10, 3215-3229.

Meredith, M. A., \& Stein, B. E. (1983). Interactions among converging sensory inputs in the superior colliculus. Science, 221, 289-291.

Meredith, M. A., \& Stein, B. E. (1986a). Visual, auditory, and somatosensory convergence on cells in the superior colliculus results in multisensory integration. Journal of Neurophysiology, 56, 640-662 
Meredith, M. A., \& Stein, B. E. (1986b). Spatial factors determine the activity of multisensory neurons in cat superior colliculus. Brain Research, 365, 350-354.

Meredith, M. A., \& Stein, B. E. (1996). Spatial determinants of multisensory integration in cat superior colliculus neurons. Journal of Neurophysiology, 75, 1843-1857.

Mey, J., \& Thanos, S. (1993). Developmental anatomy of the chick retinotectal projection. In H. P. Zeigler, \& H. Bischof (Eds.), Vision, brain, and behavior in birds. (pp. 173-194). Cambridge, MA: MIT Press.

Momose-Sato, Y., Glover, J. C., \& Sato, K. (2006). Development of functional synaptic connections in the auditory system visualized with optical recording: Afferentevoked activity is present from early stages. Journal of Neurophysiology, 96, 1949-1962.

Monk, C. S., Gunderson, V. M., Grant, K. S., \& Mechling, J. L. (1996). A demonstration of the memory savings effect in infant monkeys. Developmental Psychology, 32, 1051-1055.

Morrongiello, B. A., Fenwick, K. D., \& Chance, G. (1998). Crossmodal learning in newborn infants: Interferences about properties of auditory-visual events. Infant Behavior and Development, 21, 543-554.

Nelson, T. O. (1985). Intersensory development and Pavlovian conditioning: Stimulus selection and encoding of lights and tones in the preweanling rat. Journal of Experimental Psychology: Animal Behavior Processes, 17, 448-464.

Norton, T. T. (1974). Receptive-field properties of superior colliculus cells and development of visual behavior in kittens. Journal of Neurophysiology, 37, 674690.

Pickens, J., \& Bahrick, L. E. (1995). Infants' discrimination of bimodal events on the basis of rhythm and tempo. British Journal of Developmental Psychology, 13, 223-236.

Pickens, J., \& Bahrick, L. E. (1997). Do infants perceive invariant tempo and rhythm in auditory-visual events? Infant Behavior and Development, 20, 349-357.

Prince, C. G., \& Hollich, G. J. (2005) Synching models with infants: a perceptual-level model of infant audio-visual synchrony detection. Cognitive Systems Research, 6, 205-228.

Rafal, R., Henik, A., \& Smith, J. (1991). Extrageniculate contributions to reflex visual orienting in normal humans: A temporal hemifield advantage. Journal of Cognitive Neuroscience, 3, 322-328. 
Reynolds, G., \& Lickliter, R. (2002). Effects of prenatal sensory stimulation on heart rate and behavioral measures of arousal in bobwhite quail chicks. Developmental Psychobiology, 41, 112-122.

Rizzolatti, G., Riggio, L., Dascola, I., \& Umilta, C. (1987). Reorienting attention across the horizontal and vertical meridians: Evidence in favor of a premotor theory of attention. Neuropsychologia, 25, 31-40.

Robinson, D. L., \& Keretzman, C. (1995). Covert orienting of attention in macaques: III. Contributions of the superior colliculus. Journal of Neurophysiology, 74, 713721.

Schneider, G. E. (1967). Contrasting visuomotor function of tectum and cortex in the golden hamster. Psychologische Forschungen, 31, 52-62.

Shepperd, M., Findlay, J. M., \& Hockey, R. J. (1986). The relationship between eye movements and spatial attention. Quarterly Journal of Experimental Psychology, 38 A, 475-491.

Slater, A., Quinn, P. C., Brown, E. \& Hayes, R. (1999). Intermodal perception at birth: Intersensory redundancy guides newborn infants' learning of arbitrary auditoryvisual pairings. Developmental Science, 3, 333-338

Sleigh, M. J., Columbus, R. F., \& Lickliter, R. (1998). Intersensory experience and early perceptual development: Postnatal experience with multimodal maternal cues affects intersensory responsiveness in bobwhite quail chicks. Developmental Psychology, 34, 215-223.

Spelke, E. S. (1979). Perceiving bimodally specified events in infancy. Developmental Psychology, 15, 626-36.

Spence, C., \& McDonald, J. (2004). The cross-modal consequences of the exogenous spatial orienting of attention. In G. Calvert, C. Spence, \& B. E. Stein (Eds.). The handbook of multisensory processes (pp.3-25). Cambridge, MA: MIT Press.

Sprauge, J. M., \& Meikle, T. H., Jr. (1965). The role of the superior colliculus in visually guided behavior. Experimental Neurology, 11, 115-146.

Stein, B. E., \& Gallagher, H. L. (1981). Maturation of cortical control over superior colliculus cells in cat. Brain Research, 223, 429-435.

Stein, B. E., Labos, E., \& Kruger, L. (1973a). Sequence of changes in properties of neurons of superior colliculus of the kitten during maturation. Journal of Neurophysiology, 36, 667-679. 
Stein, B. E., Labos, E., \& Kruger, L. (1973b). Determinants of response latency in neurons of superior colliculus in kittens. Journal of Neurophysiology, 36, 680689.

Stein, B. E., \& Meredith, M. A. (1991). Functional organization of the superior colliculus. In A. G. Leventhal (Ed.), The neural bases of visual function (pp. 85110). Hampshire, England: Macmillan.

Stein, B. E., \& Meredith, M. A. (1993). The merging of the senses. Cambridge, MA: MIT Press.

Stein, B.E., Huneycutt, W.S., \& Meredith, M.A. (1988). Neurons and behavior: The same rules of multisensory integration apply. Brain Research, 448, 355-358.

Stein, B.E., Meredith, M.A., Huneycutt, W.S., \& McDade, L. (1989). Behavioral indices of multisensory integration: Orientation to visual cues is affected by auditory stimuli. Journal of Cognitive Neuroscience, 1, 12-24.

Stein, B. E., \& Meredith, M. A., \& Wallace, M. T. (1993). Nonvisual responses of visually-responsive neurons. In T. P. Hicks, S. Molotchnikoff, \& T. Ono (Eds.), Progress in brain research: The visually responsive neurons: From basic neurophysiology to behavior (pp. 79-90). Amsterdam: Elsevier.

Stein, B. E., Wallace, M. T., \& Meredith, M. A. (1995). Neural mechanisms mediating attention and orientation to multisensory cues. In M. S. Gazzaniga (Ed.), The cognitive neurosciences (pp. 683-702). Cambridge, MA: MIT Press.

Summerfield, A. Q. (1979). Use of visual information in phonetic perception. Phonetica, 36, 314-331.

Thelen, E., \& Smith, L. B. (1994). A dynamic systems approach to the development of cognition and action. Cambridge, MA: MIT Press.

Thompson, G. C., \& Masterton, R. B. (1978). Brain stem auditory pathways involved in reflexive head orientation to sound. Journal of Neurophysiology, 41, 1183-1202.

Vroomen, J., \& de Gelder, B. (2004). Perceptual effects of cross-modal stimulation: ventriloquism and the freezing phenomenon. In G. Calvert, C. Spence, \& B. E. Stein (Eds.). The handbook of multisensory processes (pp. 144-150). Cambridge, MA: MIT Press.

Wallace, M. T., Meredith, M. A., \& Stein, B. E. (1998). Multisensory integration of in the superior colliculus of the alert cat. Journal of Neurophysiology, 80, 10061010 . 
Wallace, M. T., \& Stein, B. E. (1997). Development of multisensory neurons and multisensory integration in cat superior colliculus. Journal of Neuroscience, 17, 2429-2444.

Wallace, M. T., \& Stein, B. E. (2001). Sensory and multisensory responses in the newborn monkey superior colliculus. Journal of Neuroscience, 21, 8886-8894.

Wallace, M. T., Wilkinson, L. K., \& Stein, B. E. (1996). Representation and integration of multiple sensory inputs in primate superior colliculus. Journal of Neurophysiology, 76, 1246-1266.

Wilkinson, L. K., Meredith, M. A., \& Stein, B. E. (1996). The role of anterior ectosylvian cortex in cross-modality orientation and approach behavior. Experimental Brain Research, 112, 1-10.

Zeigler, H. P., \& Bischof, H. (1993). Vision, brain, and behavior in birds. Cambridge, MA: MIT Press. 
VITA

MARK JAIME

1974

Born, Panama City, Panama

2001

B.A., Psychology

Florida International University

Miami, Florida

2003

NIH Travel Award

International Society for Developmental

Psychobiology

Annual meeting; New Orleans, LA

2004

MBRS-Research Initiative for Scientific

Enhancement

NIH Pre-doctoral Award

Florida International University

Department of Biological Sciences

2005

M.S., Psychology

Florida International University

Miami, Florida

2005

NIH Travel Award

International Society for Developmental

Psychobiology

Annual meeting; Washington, D.C.

2007

NIH Travel Award

International Society for Developmental Psychobiology

Annual meeting; San Diego, CA

\section{PUBLICATIONS AND PRESENTATIONS}

Hileman, C., Jaime, M., Schwartz, C. B., Zahka, N. E., Inge, A. P., Kojkowski, N., Coman, D., Henderson, H., and Mundy, P. C. (2007, April). Motivation and selfmonitoring as predictors of symptom severity in autism. Society for Research in Child Development. Boston, MA 
Hileman, C., Jaime, M., Schwartz, C. B., Zahka, N. E., Inge, A. P., Kojkowski, N., Coman, D., Henderson, H., and Mundy, P. C. (2007, May). Motivation and selfmonitoring as predictors of symptom severity in autism. International Meeting for Autism Research. Seattle, WA

Jaime, M. and Lickliter, R. (2005, March). The effect of prenatal exposure to temporal and spatial properties on postnatal responsiveness to spatial contiguity in bobwhite quail chicks. Comparative Cognition Society. Melborne, FL

Jaime, M., Lickliter, R. and Bahrick, L. E. (2005, November). The role of temporal synchrony in the salience of intersensory redundancy during prenatal development. International Society for Developmental Psychobiology. Washington, D.C.

Jaime, M. and Lickliter, R. (2006). Prenatal exposure to temporal and spatial stimulus properties affects postnatal responsiveness to spatial contiguity in bobwhite quail chicks. Developmental Psychobiology, 48, 233-242.

Jaime, M., Lickliter, R. and Bahrick, L. E. (2006, June). Exploring the temporal boundaries of intersensory redundancy during prenatal development. International Conference on Infant Studies. Kyoto, Japan

Jaime, M., Bahrick, L., and Lickliter, R. (2007, October). Audiovisual temporal synchrony facilitates prenatal perceptual learning in bobwhite quail embryos. International Society for Developmental Psychobiology, San Diego, CA.

Lopez. J.P., Jaime, M., and Lickliter, R. (2007, October). Bobwhite quail neonates track human eye gaze to direct their avoidance behavior. International Society for Developmental Psychobiology, San Diego, CA. 